\title{
Evaluation of the Therapeutic Effect of MNRI Reflex Neuromodulation on Children Diagnosed with Autism Based on Reflex Assessments, QEEG Analysis and ATEC Questionnaire
}

\author{
Katarzyna Novak ${ }^{*}$, Piotr Sobaniec ${ }^{1}$, Wojciech Sobaniec ${ }^{1}$, Nelli Akhmatova², and Patty Shackleford ${ }^{3}$ \\ ${ }^{1}$ Neurology and Child Rehabilitation, Department of the Medical University of Białymstok of Poland, Poland \\ ${ }^{2}$ Mechnikov Institute of Vaccines and Serums, Moscow, Russian Federation, Russia \\ ${ }^{3}$ Svetlana Masgutova Education Institute, Orlando, FL, USA
}

*Corresponding author: Katarzyna Novak, Neurology and Child Rehabilitation, Department of the Medical University of Białymstok of Poland, Poland, Tel: 6082359084; E-mail: sally@masgutovafoundation.org

Received: 27 Feb, 2020 | Accepted: 15 Apr, 2020 | Published: 25 Apr, 2020

Citation: Nowak K, Sobaniec P, Sobaniec W, Akhmatova N, Shackleford P (2020) Evaluation of the Therapeutic Effect of MNRI Reflex Neuromodulation on Children Diagnosed with Autism Based on Reflex Assessments, QEEG Analysis and ATEC Questionnaire. J Neurol Neurobiol 6(2): dx.doi. org/10.16966/2379-7150.165

Copyright: (c) 2020 Nowak K, et al. This is an open-access article distributed under the terms of the Creative Commons Attribution License, which permits unrestricted use, distribution, and reproduction in any medium, provided the original author and source are credited.

\begin{abstract}
Introduction: The hypotheses of neurodevelopmental causes of Autism Spectrum Disorder (ASD) are still to be discovered. Recent studies show some reasons to include abnormalities in the development of the brain and central nervous system. The MNRI-Masgutova Neurosensorimotor Reflex Neuromodulation Program uses the concept of rehabilitation of early sensory-motor reflex patterns, or units of the nervous system, to optimize the brain development of children with ASD and also with other neurological disorders.
\end{abstract}

Objective: The children were evaluated for A) reflex patterns, B) brain maps and C) social-behavioral-cognitive traits (6-11 year-old children [n=30]).

Methods: Evaluation methods of A) MNRI Reflex Integration Assessment, B) QEEG Quantitative Analysis, and C) the Autism Treatment Evaluation Checklist (ATEC) questionnaire for parents/guardians filled out prior to and after the MNRI training program used at intensive 8-day Family Conferences.

Results: All three evaluation methods showed substantial positive changes in children with ASD in several important developmental areas after MNRI treatment, particularly in:

1. MNRI Reflex Assessment: Remarkable progress in $33.33 \% / 10$ out of 30 patterns took place on a statistical significance level ( $p>0.01$ ), and in $36.67 \% / 11$ close to statistical significance, which is supported by qualitative analyses.

This study shows that the neurodevelopment and overall functioning of individuals with neurodeficits such as Cerebral Palsy (CP) is not static in pathologies and could be successfully improved with the MNRI form of neuromodulation treatment.

2. Brain Wave spectrum: $76.67 \%$ (out of 30 ) children displayed a reorganization of spontaneous brain electrical activity observed in the increase in alpha frequency range and decrease in fast beta activity in parietal and temporal locations. This may be connected with the positive and stable therapeutic effect of the MNRI method on motor disorders originating in the central nervous system.

\section{The ATEC test demonstrated changes in the following life skills:}

1) Speech, language and communication-changes are noted in $31 \%$ of children (statistically significant level), of $31 \%$-close to statistical significance;

2) Socialization-changes are noted in $48 \%$ of children (statistically significant level), of $22 \%$-close to statistical significance.

3) Sensory Sensation and Cognitive Awareness-changes are noted in $38 \%$ of participants (statistically significant level), of $16 \%$-close to statistical significance.

4) Health, Physical, Behavior -Sensory Sensation and Cognitive Awareness-changes are noted in $59 \%$ of participants (statistically significant level), of $10 \%$-close to statistical significance.

Conclusion: This study has demonstrated the normalization effect of the MNRI Program on: a) development of reflex patterns b) brain wave spectrum (QEEG), both resulted in c) improvement of most challenging areas of life and learning of children with ASD-speech and communication, socialization, sensory and cognitive awareness, physical health and behavioral-emotional regulation proving the value of the MNRI restorative concept for dysfunctional reflex circuits.

Significance of Results: The results of this study demonstrates that's the neuromodulating and normalizing effect on neurodevelopment of individuals with ASD-corrective and preventive-can be enhanced with this unique novel form of MNRI neuromodulation treatment targeting the paving and rerouting nerve pathways to bring them into a state of proper neurophysiological functioning to improve the excitation-inhibition processes of the nervous system, increasing the stress resilience and neuroplasticity.

Keywords: Autism spectrum disorder; Neuromodulation; MNRI; Cognitive; Reflex integration; Regulation 


\section{Introduction}

\section{Autism spectrum disorder: Definition and symptoms}

In 1911, the Swiss psychiatrist Eugen Bleuler, considered an expert on schizophrenia, suggested the term "autos" the Greek word autos $[1,2]$ to identify the most severe schizophrenics as autistic. He based this on a small group of severe schizophrenics, who were observed to be withdrawn from social life and did not react to the attempt to make contact as autistic. In 1973, Kanner L [3], an American doctor recognized as the creator of pediatric psychiatry, suggested the definition of early childhood Autism, included the Disorder Syndrome (ASD), to be a severe disorder manifested by verbal and non-verbal communication and extreme stereotypical behaviors. He created this autism definition after observing a group of 11 children who displayed unique social relationships, isolated, unable to create emotional ties even with their nearest devices $[1,2]$.

The hypotheses of neurodevelopmental causes of autism are still unambiguous and not confirmed [1,2]. The most frequent are the causes of abnormalities in the development of the central nervous system since the prenatal period [2]

In individuals with autism, several brain structures and functions have been detected in the temporal-occipital area, amygdala and frontal lobes to be deviated from the norm. All these structures are responsible for social interactions. Other brain areas that are different from the norm included: the construction of dendrites and the limbic system that effect social interaction are different compared to norm $[1,4,5]$. Also, differences are noted in the brain size and weight, physical height and overall growth compared to 'typical' children of the same age. It is also noted that children with ASD are born with normal brain mass, and from the fourth month on to the first few years of life their brain mass increases and becomes more intense than in their peers [6,7]. Although there is much information on the development of the young children with ASD, there still is no a comprehensive picture of overall symptoms for this disorder. Research suggests that the risk factors involved in this developmental delay include genetic (chromosomal) and environmental (internal and external) factors $[6,8]$. Genetic, metabolic, viral and teratogenic (pathological) factors also affect the disorder. The occurrence of the global delays that is later diagnosed as autism is associated with chromosomal abnormalities. The nature of autism in combination with abnormal development and atypical brain functions leads to the assumption that it is caused by a genetic susceptibility and/or the effect of environmental factors, e.g. biochemical, which have not yet been discovered [9].

The clinical picture of autism is heterogeneous and also affected by other diseases and symptoms. Leo Kanner suggests that there are 11 precursors for the development of autism [3]:

- Inability to develop social relations.

- Stereotypical activities while playing/in games.

- Occurrence of disorders in infancy.

- Lack of speech development or inability to adequately use communication skills.

- The ability to automatically memorize words without comprehension.

- Tendency for conservatism/invariability.

- Lack of imagination.

- Delays in language development.

- Incorrect usage of pronouns.
- Echolalia.

- Attacks of anxiety and aggression.

Additional clinical observations noted sensory hypersensitivity in these children including reluctance to touch; auditory, visual and olfactory hypersensitivity; hyperactivity disorder; difficulty in expressing emotions which may be manifested by aggression to self and others $[10,11]$.

Overall, autism as a developmental disorder which requires a full multi-profile diagnosis to determine the child's neurodevelopment, level of functionality, and specific direction/s for the appropriate individualized therapy. The diagnosis should be determined based on the following areas $[9,12]$ :

a. Communication: The stage of speech development, number of sounds and words, ability to combine sounds or words together, use of sounds and words to signal needs, use of correct grammar, rule out echolalia, following simple commands/instructions, and ability to understand temporal interactions/relations.

It is also important to determine their ability to use special gestures, pictograms, and/or use of direct interaction with others to get wants and needs met.

b. Social development: Tendency to avoid or initiate visual and tactile clues for personal contact; reactions to people, animals and objects; and level of motor development.

c. Specific features of ASD disorder: Resistance to ongoing changes, inability to recognize people, resist attempts to imitate, inability to complete daily living/self-service activities, inability to maintain consistent orientation, hyper/hypo reaction to environmental stimuli, inability for adequate time management, inability to focus attention.

d. Reflex patterns: The level of reflex patterns integration and finding the key for developing a correct plan for improving reflex integration to assist overall positive neurodevelopment in context of the entire neurodevelopment. Difficulties that are observed in children with the ASD include the lack of communication, sensory hyperactivity, psychomotor hyperactivity or tendency for aggression, sensory processing deficits and in appropriate reflex patterns.

To develop an effective rehabilitation plan to assist the child with ASD a multi-profile an effective, a multi-profile rehabilitation plan based on the integration of reflexes and stimulation of the proper primary reflex mechanisms must be developed.

\section{MNRI Neuromodulation Intervention}

The creator of the MNRI (Masgutova Neurosensorimotor Reflex Integration) Program, Svetlana Masgutova, is a graduate of the General and Developmental Psychology Research Institute of the Russian Education Academy. In 1998 she was awarded a Ph.D. degree in developmental and educational psychology, and four years later she was awarded the title of Associate Professor in Psychology [13]. In her research Dr. Masgutova referred to the works of L. Vygotsky, I. Bozovich, I. Dubrovina and N. Tolstykh, the authors of over 200 scientific and practical works. Dr. Masgutova collaborated with many research and scientific centers, including the Early Intervention Department of the Wroclaw Medical Academy. She received a specialization in the field of clinical speech pathology and conducted research studies at the Russian Academy of Education.

Since 1989 Dr. Masgutova has been conducting clinical observations and research on the influence of primary motor and reflex patterns on various aspects of development, including cognitive processes [13]. 
The MNRI program implements the concept of correction and integration of dysfunctional reflex patterns, motor skills [14], and is directed, particularly, at the integration of:

-Sensorimotor aspects of a reflex pattern including how to secure proper neurological functioning within a specific "reflex circuit" [15-18]:

-Reflex patterns for intentional motor activity;

-Reflex patterns for motor consciously controlled abilities and skills;

-Reflex patterns for physical, cognitive, and behavioral processes.

The MNRI method supports the development of children and adults. Techniques are natural, safe and can be easily taught to parents of children with challenges to use in a home program as well as helping personnel and professionals who work with this population. This method does not require large expenditures and can be used as a complementary program with other therapy modalities [19].

The method includes the following programs [20],

- MNRI: Integration of Dynamic and Postural reflexes,

- MNRI: Oral-Facial Reflex Integration (Level 1 and 2),

- MNRI: NeuroStructural Reflex Integration,

- MNRI: NeuroTactile Integration,

- MNRI: Archetype Movement Integration,

- MNRI: Visual-Auditory Reflexes Integration,

- MNRI: Lifelong Reflex Integration,

- MNRI: Dysfunctional and Pathological Reflex Repatterning and Integration,

- MNRI: Birth and Post-Birth Reflex Integration,

- MNRI: Reflexes Integration for Post-Trauma and PTSD,

- MNRI: Reflex Integration and Basal Ganglia,

- MNRI: Reflex Integration for Stress Hormones Regulation,

- MNRI: Proprioceptive-Cognitive Integration,

- MNRI: Dyslexia and Reflex Integration,

- MNRI: Art-Therapy and Reflex integration.

All programs are used by professionals certified in the MNRI Program [www.MasgutovaMethod.com].

\section{Objective of the study}

The evaluation of the effect of the MNRI Program on the progress of children with ASD based on quantitative QEEG analysis, MNRI Reflex Assessment, and the ATEC test.

\section{Materials and Methods}

\section{Materials}

During 2015-2018, the International Dr. Svetlana Masgutova Institute in Poland and the Svetlana Masgutova Educational Institute in the US organized a research project based on brain mapping children with autism before and after comprehensive MNRI Family Rehabilitation Conferences for children with autism.

This study included 30 participates including: 3 girls (10\%) and 27 boys (90\%) aged 6-11 years old with pediatric autism diagnosed according to ICD-10 criteria (F84.0). The average age of the participates was 11 years $(\mathrm{M} \pm \mathrm{SD}=8.5 \pm 2.8)$. All the children in this study attended the MNRI Family Conference/Camps and received 6 hours of the MNRI therapy for 8 days.

\section{Criteria for inclusion in the study were:}

- Children with diagnosed autism according to ICD-10-(F84.0),

- Aged 6-11 years,

- Ability for cooperation during brain map testing,

- The possibility of active participation in an 8-day rehabilitation conference.

Exclusion criteria from participation in the study:

- Failure to carry out the initial assessment, evaluation questionnaire or EEG registration,

- Head injury medical history, epilepsy,

- Taking/use of neurological or psychiatric drugs,

- Excessive level of hyperactivity or aggression,

- Mental retardation to a deep degree,

- Contraindications from attending the Conference from a physician.

\section{Methods}

Stage One of Research (duration 1-2 days): The first stage included assessing children with ASD using EEG, QEEG quantitative analysis, MNRI reflex Assessment, and parents/guardians filling out the Autism Treatment Evaluation Checklist (ATEC) questionnaire as part of the research.

Stage Two (duration8 days): The second stage of the project was the Conference/rehabilitation process using the MNRI programs. Each patient underwent 6 sessions of 50 minutes a day. Classes were divided between $8.50-12.40$ and 14.00-18.00, with a 1.5 h. lunch break.

The first half of the Conference is 4 days, followed by one day off and another 4 therapeutic days. Most often, sessions were conducted by a MNRI Core Specialist. Only MNRI techniques were used during the research time period.

Stage Three (duration 1-2 days): The third stage included having study subjects post-tested on the EEG examination, QEEG quantitative analysis, MNRI Reflex Assessment, and parents/guardians again filled the ATEC questionnaire on the last day of the MNRI Conference.

In total, 3,234 hours of therapy and over 950 diagnostic and analytical hours were carried out with the Study group.

\section{Methods: Evaluation and rehabilitation tools}

MNRI reflex assessment: The MNRI Assessment of dynamic and postural reflexes defines 30 exemplary reflex schemas, oriented at the following criteria

- The proper reaction for a given stimulus in relation to the age of a child and the neurophysiological norm: certain sensory stimulus causes a specific motor response.

- Proper direction of motor or postural response (activity of motorproprioceptive system; motor neuron activity).

- Proper intensity/muscle tone (norm, hyper-active, hypo-activity, a-reflexive response).

- Latency-reaction time.

- Symmetry in the reaction. 
Table 1, The Reflex Assessment's main orientation is determining if feedback responses within the reflex physiological circuits are proper to the given genetic motor pattern [21]. The level of maturity of a reflex affects the brain, which influences, in its turn, the development of the motor, social, emotional skills, and other factors [22,23]. Statistical analysis of the results of Reflex Assessment was carried out to create Reflex Profiles for children with ASD to identify strategies for neurodevelopment corrective work for them. This analysis was also accomplished after 8 days of MNRI corrective processes at the neurosensorimotor rehabilitation camps/clinics.

The Reflex Assessment plays a crucial role for learning timely information (possible disorders) on the child's state of reflex development and gives information on how to develop an organized, timely preventive or corrective work to support neurodevelopment [19].

Quantitative QEEG analysis: Quantitative QEEG analysis refers to an Electroencephalographic Examination (EEG) which is a noninvasive assessment of brain functions $[24,25]$. The bioelectrical activity of the brain is the averaged activity of much postsynaptic potential, created as a result of synchronous stimulation of nerve cells. An EEG allows the bioelectrical activity of the brain to be recorded with the help of electrodes placed on the surface of an individual's head, and the information is gathered and presented in the form of brain wave plots [26]. The reference (single-pole) assembly was used in the study as the most popular montage because of its wide use in different countries that was enabling the comparative analysis of research tool choice and extending the possibilities of the results interpretation.

QEEG quantitative analysis uses computational methods, eg: Fast Fourier Transformation (FFT) or wave spectrum analysis which provides statistical data and discrete variables of the EEG signal in the frequency domain [24]. One of the parameters commonly analyzed in the QEEG analysis is the power spectral density for individual frequency ranges [27].

The method of use for EEG registration was a 24-channel Brain master Discovery device with automatic Electrocap, with electrodes located in accordance with the international electrode arrangement system 10-20 (Fp1, Fp2, F3, F4, F7, F8, Fz, C3, C4, Cz, T3, T4, T5, T6, $\mathrm{P} 3, \mathrm{P} 4, \mathrm{Pz}, \mathrm{O} 1, \mathrm{O} 2$, and two reference electrodes attached to the ear

Table 1: Criteria for MNRI Assessment of Reflex Maturity in Points (1-20).

\begin{tabular}{|c|c|c|c|}
\hline \multicolumn{4}{|c|}{ Criteria of Level of Reflex Patterns Development } \\
\hline & Normal Function & & $\begin{array}{l}\text { Dysfunction/ } \\
\text { Pathology }\end{array}$ \\
\hline Points & Level of reflex integration & Points & $\begin{array}{l}\text { Level of reflex } \\
\text { dysfunction }\end{array}$ \\
\hline 20 & Full/Complete integration & $10-11.75$ & $\begin{array}{c}\text { Marginal pathology } \\
\text { and dysfunction }\end{array}$ \\
\hline 18-19.75 & Mature and integrated & $8-9.75$ & $\begin{array}{l}\text { Incorrect, light } \\
\text { dysfunction }\end{array}$ \\
\hline $16-17.75$ & $\begin{array}{c}\text { Correctly developed- } \\
\text { normal }\end{array}$ & 6-7.75 & Dysfunction \\
\hline 14-15.75 & $\begin{array}{c}\text { Functional, but low level } \\
\text { of development }\end{array}$ & $4-5.75$ & Severe dysfunction \\
\hline $12-13.75$ & $\begin{array}{l}\text { Functional, but very low } \\
\text { level of development }\end{array}$ & 2-3.75 & Pathology \\
\hline 10-11.75 & $\begin{array}{l}\text { Marginal pathology and } \\
\text { dysfunction }\end{array}$ & $0-1.75$ & Severe pathology \\
\hline
\end{tabular}

lobes A1, A2) [28]. The caps were used in this study to ensure high repeatability of electrode location, easy assembly and convenience, which is important especially, in this case evaluating children with autism, who usually show disturbed sensory sensation. The sizes of the caps were chosen according to the circumference of the subject's head. $\mathrm{Nu}$-prep abrasive paste was used to prepare the scalp and ears for the electrodes, and the electrodes were filled with Electro-gel conductive gel. The acceptable impedance level for all electrodes was below $10 \mathrm{k} \Omega$. Multiple measurements with open and closed eyes were recorded for a minimum of 5 minutes for each stage. A sample value of 256 samples/ sec; filters: $\mathrm{LP}=0.3 \mathrm{~Hz}, \mathrm{HP}=70 \mathrm{~Hz}$ and $50 \mathrm{~Hz}$ mains filter [27].

NEUROGUIDE software [29] was used to analyze each QEEG, containing normative results developed on the age group of 2-82 years, $\mathrm{N}=470$ children and $\mathrm{N}=155$ adults. The recorded EEG signal was subjected to automatic detection eliminating motion artifacts, voltage, blinking eyes and other, and then checked by the EEG technician to verify correctness and to supplement the automatic elimination of artifacts. The purified EEG record was subjected to FFT analysis. Using statistical analysis, the data of each child was compared with the results of the database in norm corresponding to a specific age. The absolute values of the signal power for the following frequency bands were selected for statistical analysis [27]:- Delta $(0-4 \mathrm{~Hz})$, -Theta (4-8Hz), -Alpha $(8-12 \mathrm{~Hz})$, -Beta $(12-25 \mathrm{~Hz})$, -LoAlpha $(8-10 \mathrm{~Hz})$, -HiAlpha $(10-12 \mathrm{~Hz})$, -Beta1 $(12-15 \mathrm{~Hz})$, -Beta2 (15-18 Hz), -Beta3 $(18-25 \mathrm{~Hz})$, -High Beta $(25-30 \mathrm{~Hz})$. To evaluate the changes in the bioelectric activity of the brain, the analysis of the absolute power Z-score parameter for all 19 leads was used.

Statistical methods: The ANOVA test for statistical analysis (IBM SPSS Statistics Grad Pack 22.0) was used for determining the values of reflex patterns changes between 0 and 1 based on a summary of 30 values scored from 0 to 20 for each individual. Mean values were compared prior to and after 8 days of going through the MNRI program. Results were approached as statistically significant at $\mathrm{p}<0.001$ and not significant at $p>0.05$. The statistical evaluations were also performed by the Mann-Whitney U-test and additionally by statistical (version 6.0; Stat Soft Inc, Tulsa, OK, USA). P values $(\mathrm{M} \pm \mathrm{SD}$ ) less than 0.001 in the same way were evaluated as significant at $p<0.001$ and not significant at $\mathrm{p}>0.05$.

The brain mapping analysis has been using the similar statistical logics: as a part of the descriptive analysis of the real variable, the median values were compared to a normal distribution of mean values and standard deviations. For binary or qualitative attributes the percentages for different numerical values was used. Wilcoxon test was utilized to compare the results obtained before and after the study within the median comparison. If the variables were subject to normal distribution and analysis, then a mean comparison was made using the t-test for dependent variables. Comparison of results in independent groups was carried out using the t-test within the normalization assumptions and homogeneity of variants or nonparametric tests for independent to do a deeper level of the means comparison. The Levene test was used to check the homogeneity of the use variants. Significance level accepted at 0.05 has been corrected for correlations between variables. Due to the fact that the data can be treated as data collected clusters, additional methods applied to the data forming clusters.

\section{Autism Treatment Evaluation Checklist (ATEC)}

ATEC [30] is a standardized questionnaire developed by The Autism Research Institute, USA). It is a simple but effective tool to measure the effectiveness of various treatments for autism. The maximum number

Citation: Nowak K, Sobaniec P, Sobaniec W, Akhmatova N, Shackleford P (2020) Evaluation of the Therapeutic Effect of MNRI Reflex Neuromodulation on Children Diagnosed with Autism Based on Reflex Assessments, QEEG Analysis and ATEC Questionnaire. J Neurol Neurobiol 6(2): dx.doi.org/10.16966/2379-7150.165 
of points is the result of 179 questions-the higher the score, the higher the degree/severity of the disease [31].

The tool has no limitations based on age and takes about 20 minutes to fill out and complete. Questions are grouped into four categories corresponding to the following areas $[31,32]$ of:

- Speech/Language/Communication communication),

(Speech/language/

- Sociability (Socializing),

- Sensory/Cognitive Awareness (Awareness of the senses/cognitive processes),

- Health/Physical/Behavior (Physical Health/Behavior).

\section{MNRI Rehabilitation Modules}

This study provided each child with autism a specific program based on their personal assessment results on sensory-motor reflex circuit patterns functions and brain mapping. MNRI practitioners applied MNRI techniques over an eight-day period (four days on, one day off, four days on) with the day in the middle being for rest, with no application of techniques. Each of the technique application days had six 50-minute sessions of work. The MNRI techniques drawn upon for this work included the following:

a) MNRI Reflex Repatterning

b) MNRI Neurostructural Reflex Integration and Immune System

c) MNRI NeuroTactile Integration

d) MNRI Archetype Movements Integration

e) MNRI Breathing Reflex Integration

f) MNRI Stress and Trauma release

g) MNRI Proprioceptive-Cognitive Integration

h) MNRI Oral-Motor/Visual-Auditory Reflexes Integration.

Each of the above treatment sessions was focused on a specific process of neurodevelopment.

a) MNRI Reflex Repatterning aims at strengthening and improving the neurological functioning and connectivity between the sensory and motor neurons of a specific reflex $[19,33,34]$ that influence the sensory-motor mile stones, motor programming, planning and control, and also cognitive skills [19,35-37]. Supporting these individual reflex patterns allows for the improvement of the functioning of disturbed/ impaired reflex patterns and stimulation of their electrical-mediator regulatory mechanisms. An improperly functioning reflex interferes with the development of controlled motor skills, blocks an adequate perception, rational thinking and behavior control.

b) Neurostructural Reflex and Immune System Integration focuses on improving the functions of reflexes responsible for postural control, spine flexibility, abdomen, neck and limbs musculature tone regulation, and the release of core tendon guard (creating positive protection and the feeling of being secure). Immunomodulatory effects were aimed at improvement of functions in T-1 immunity, cytokinesis, CD-4, CD-8 and other immune cell functions, anti-inflammatory effect, and regulation of immunoglobulins (IgE, IgG and others) [38-40]. It works directly with the body structure and the mechanism of symmetry which is essential for the operative functioning of reflex patterns. The main goal is normalization of muscle tone and activation of proprioceptors (deep sensation and pressure receptors) for facilitation of the reflex patterns. c) Archetype Movements Integration focuses on the enhancement of the primary biomechanics of motor patterns (extension, flexion, rotation, stretching-compression, and other) giving support for structural aspects of numerous reflex patterns, development of automatic and consciously learned motor abilities and skills; also postural and motor control, with secondary improvement in the speed of perception, focusing, and memory in sensory-motor integration and cognitive functions [41]. This program is based on the use of "inherent biomechanics of the motor programs" that accompany a human throughout their lives. The archetypal motor patterns activate functions such as brain-motor programming, planning, control of the physical body, and goal-orientation for hands-on tasks.

d) Breathing Reflex Integration focuses on the regulation and normalization of Breathing Reflex patterns and the residual volume of the lungs for normal breathing and on creating protection and survival [14].

e) Stress and Traumatic Stress Release focuses on reflex patterns that can impact the HPA stress-axis for letting go of past negative stressors and traumas, and for trauma normalization by activation of stress hormones and neurotransmitter regulation [40,42].

f) Proprioceptive-Cognitive Integration focuses on improving proprioceptive-vestibular (balance) system related reflexes for support of postural and motor control, with secondary improvement in motorcognitive functions.

g) Oral-Motor/Visual-Auditory Reflexes Integration focuses on improving oral-motor articulation and speech abilities, as well as visual and auditory functions [43]. This program was designed to integrate oral-facial, motor, visual and auditory reflexes to regulate muscle tone and facilitate the complex coordination needed for speech, eating and expressing emotions. It also facilities the teaming of the visual and auditory reflexes and improves the natural functioning of vision and hearing in order to optimize growth, development and learning.

h) MNRI: Repatterning and Integration of Dysfunctional and Pathological Reflexes focuses on neuromodulation or correction exercises and procedures for dysfunctional and pathological motor and reflex patterns.

\section{Results and Interpretation}

\section{MNRI reflex assessment and Progress in Reflex Patterns}

The results of comparative analysis of MNRI Reflex Assessment in children with ASD aged 6-11(n=30) done before and after the MNRI intervention show remarkable progress in reflex patterns, and particularly statistically significant changes in $33.33 \% / 10$ out of 30 patterns [Table 2], and in $36.67 \% / 11$ out of 30 patterns close to statistically significant, which were noted by parents and MNRI professionals as improvements in sensory-motor integration and motor abilities and skills.

The analysis of progress of reflex patterns showed significant improvements in children's sensory-motor abilities and skills, and particularly:

-Core Tendon Guard: The average was 12.97 points prior the MNRI Program and increased to 13.53 points $(p<0.0001)$ after by improving the symmetry of the torso, postural and head righting control, reduction of sensory hypersensitivity within the torso area the HPA stress-axis; regulation of protective behavior, ability to focus better, convergence, muscle tone regulation, and postural control, and reduction of anxiety, anger and frustration. 
Table 2: Results of comparative analysis of MNRI Reflex Assessment in children with ASD aged 6-11( $n=30$ ) before and after MNRI intervention.

\begin{tabular}{|c|c|c|c|c|c|c|c|c|c|c|c|c|c|}
\hline \multirow{2}{*}{$\begin{array}{l}\text { Reflex Patterns of Children } \\
\text { aged 6-11 y.o. }\end{array}$} & \multicolumn{6}{|c|}{ Before the MNRI Therapy Intervention } & \multicolumn{7}{|c|}{ After the MNRI Therapy Intervention } \\
\hline & $\mathrm{n}_{1}$ & Median $_{1}$ & Average $_{1}$ & Error. St ${ }_{1}$ & $\operatorname{Min}_{1}$ & $\operatorname{Max}_{1}$ & $\mathrm{n}_{2}$ & Median $_{2}$ & Average $_{2}$ & Error. st $_{2}$ & $\operatorname{Min}_{2}$ & $\operatorname{Max}_{2}$ & $\mathrm{p}$ \\
\hline $\begin{array}{l}\text { Assessed Reflexes / Number } \\
\text { of Particip. }\end{array}$ & 30 & & & & & & 30 & & & & & & \\
\hline $\begin{array}{l}\text { Core Tendon Guard (HPA } \\
\text { stress-axis resp) }\end{array}$ & 30 & 12.375 & 12.97 & 1.78 & 9.25 & 16.75 & 30 & 13.75 & 13.53 & 1.75 & 10 & 17.25 & $<0.0001^{*}$ \\
\hline Robinson Hands Grasp & 30 & 11.25 & 10.25 & 3.09 & 5.25 & 17 & 30 & 11.75 & 10.94 & 2.89 & 6 & 17 & $<0.0001^{*}$ \\
\hline Hands Pulling & 30 & 9.25 & 10.52 & 3.11 & 6 & 17.25 & 30 & 11.375 & 11.30 & 3.02 & 6 & 17.25 & $<0.0001^{*}$ \\
\hline Babkin Palmomental & 30 & 10.5 & 10.12 & 3.57 & 4.75 & 17.25 & 30 & 11.75 & 10.68 & 3.46 & 5 & 17.25 & $<0.0001^{*}$ \\
\hline Babinski & 30 & 9.5 & 9.64 & 2.61 & 5.25 & 16.25 & 30 & 10.125 & 10.38 & 2.46 & 5.5 & 16.25 & $<0.0001^{*}$ \\
\hline Leg Cross-Flexion/Extension & 30 & 11.25 & 11.33 & 3.03 & 5.25 & 16 & 30 & 11.125 & 11.96 & 2.81 & 7.25 & 16 & $<0.0001^{*}$ \\
\hline Hands Supporting & 30 & 10.25 & 10.17 & 2.60 & 6 & 17 & 30 & 10.625 & 11.12 & 2.35 & 7.5 & 17 & $<0.0001^{*}$ \\
\hline Spinal Perez & 30 & 10.375 & 10.59 & 2.80 & 6 & 16.75 & 30 & 11 & 11.43 & 2.60 & 6.25 & 16.75 & $<0.0001^{*}$ \\
\hline $\begin{array}{l}\text { Abdominal Sleep Posture } \\
\text { (Calming down) }\end{array}$ & 30 & 8.75 & 9.28 & 2.63 & 5.25 & 16.25 & 30 & 9.875 & 10.18 & 2.64 & 6 & 16.25 & $<0.0001^{*}$ \\
\hline Pavlov Cognitive Orientation & 30 & 9 & 10.18 & 2.82 & 6.5 & 16.75 & 30 & 9.875 & 10.89 & 2.83 & 7 & 16.75 & $0.0002 *$ \\
\hline
\end{tabular}

Robinson Hands Grasp: The average was 10.25 points prior to MNRI intervention and increased up to 11.30 points $(\mathrm{p}<0.0001)$ after by assisting with gross, medium and fine manual skills, hand-eye coordination and space-time orientation for speech, fine motor, handeye coordination, and muscle tone regulation.

-Hands Pulling: The average was 10.52 points prior to MNRI intervention and increased to 11.38 points $(\mathrm{p}<0.0001)$ after by assisting muscle tone regulation, symmetry in gross manual skills, improvement of head-torso coordination, reduction of hypersensitivity in the area of chest communication, hand-eye coordination; visual skills for convergence-divergence, near-far, 3D vision and visual-auditory coordination, and speech.

-Babkin Palmomental: The average was 10.12 points prior to MNRI Intervention which increased to10.68 points $(\mathrm{p}<0.0001)$ after by improving development of eating functions (chewing, swallowing); hand-eye coordination, voice modulation, articulation and speech.

-Babinski: The average was 9.64 points prior to the MNRI which increased to 10.38 points $(\mathrm{p}=0.0001$ ) after by assisting their grounding and stability in standing and walking; their sense of protection of their body; control and mobility of joints; crawling, walking skills (less toe walking) and a sense of balance.

-Leg Cross-Flexion/Extension: The average was 11.33 points prior to MNRI which increased to 11.96 points $(\mathrm{p}<0.0001)$ afterward by improving the development of cross-lateral motor programming and control, limb differentiation, walking, balance/equilibrium, and grounding/stability.

-Hands Supporting: The average was 10.17 points prior to MNRI Program which increased to 11.12 points $(\mathrm{p}<0.0001)$ after by strengthening their hand-head-torso coordination, mobility of the arms, ability for holding and handling of objects in mid-distance space, regulation of protective responses (behavior in a new environment and with new people), symmetry in gross manual skills, and hand-eye coordination, communication skills, reduction of anxiety, anger and frustration.

-Spinal Perez: The average 10.59 points prior to the intervention increased up to 11.43 points $(\mathrm{p}<0.0001)$ assisting the developing postural control, head righting, and focusing, stress regulation and detoxification of the body.

-Abdominal Sleep Posture: The average 9.28 points prior to the intervention increased up to 10.18 points $(\mathrm{p}<0.0001)$ assisting with the calming down the system, sleep patterns and stress release; detoxification of the body; reduction of anxiety, anger and frustration.

-Pavlov Cognitive Orientation: The average was 10.18 points prior to MNRI which increased to 10.89 points $(\mathrm{p}<0.0001)$ afterward by improving muscle tone activity, development of curiosity and goal orientation; skills for focusing, advanced 'head and eye' links, reading, writing, drawing and other fine motor activities. The results also were noted in increased motivation for communication, which is an important factor influencing development of children with ASD [44].

Comparative analysis of results prior and after MNRI intervention for children with ASD aged 6-11 $(n=30)$ presented below show the progressive dynamic in reflex patterns in graph expression [Figure 1].

The range of other reflex patterns showed improvement near to statistical significance ( $36.67 \% / 11$ out of 30 patterns), and, included:

Foot Tendon Guard which assists in developing a sense of protection and focus; improves postural control; improves muscle tone regulation; and assists in stress release.

Spinal Galant which assists with HPA stress-axis regulation, postural control, head righting, focusing and peripheral vision; helps improve eye tracking and comprehension.

Balancing which improves static and dynamic equilibrium and postural control of the whole body.

Flying and landing assists in postural control, head righting, focusing and stress hormones release; increases endorphin production for improved mood and mental state.

Moro which assists in HPA stress-axis regulation improves protection, fear, focus and postural control; improves muscle tone regulation, stress release and convergence of eyes.

Automatic Gait which assists with developing matured walking; improves goal orientation, sequential and gross 
motor coordination; helps improve multi-tasking and eye-leg coordination.

- Trunk Extension which improves balance, postural control, goal orientation; assists with focusing including peripheral vision and eye-leg coordination.

- Grounding which assists with stability, crawling, and walking; improves postural control and eye-head leveling.

- Symmetrical Tonic Neck (STNR) which assists with postural control, head righting and goal orientation; improves focusing, peripheral vision, functional visual-auditory skills, eye tracking, reading, writing, and drawing.

- Spinning which assists with postural control, differentiation for head-trunk motion differentiation, space-time orientation and rotation skills.

- Locomotion which assists with postural control, differentiation for head-trunk-leg motion, space-time orientation and rotation skills.

\section{QEEG results and progress in brain maps}

Electrodes activity recording from mid-frontal lobe areadorsolateral prefrontal cortex: F3 (Left)-F4 (Right)-Area 46: Main Functions of these areas: executive functions-cognitive processes, working memory, cognitive flexibility, and planning. Additional functions: Drive, attention and motivation, spontaneity in language and behavior, desire to do things for themselves and/or for others [45-48].

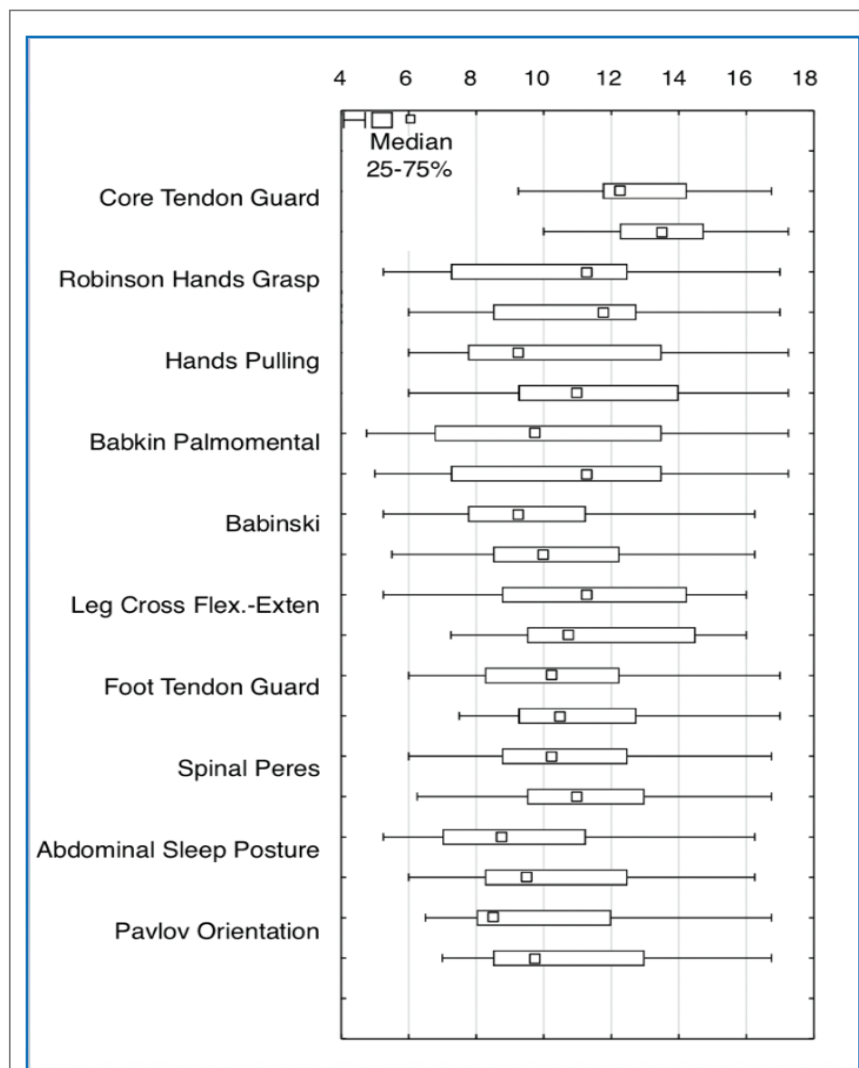

Figure 1: Comparative analysis of the results from before and after MNRI intervention on the reflex patterns for children with ASD aged $6-11(n=30)$.
Mid-frontal electrodes recorded the activity mainly from frontal brain lobes region (F3-F4), and are presented in the figures $2 \mathrm{~A}$ and $2 \mathrm{~B}$.

The data in figures $2 \mathrm{~A}$ and $2 \mathrm{~B}$, and table $2 \mathrm{~A}$ show that the median distribution for individual frequency bands of EEG waves $(1-25 \mathrm{~Hz})$, for the F4 electrode (right area of the frontal lobes of the brain), improved significantly between the pretests before the MNRI intervention and after the 8 days of intervention. Statistically significant reduction of EEG activity for Delta (1-4 Hz), Theta $(4-8 \mathrm{~Hz})$, Alpha $(8-12 \mathrm{~Hz})$, Alpha1 (8-10 Hz), Alpha2 (10-12Hz), Beta1 (12-15 Hz), also Beta2 $(15-18 \mathrm{~Hz})$ and Beta3 $(18-25 \mathrm{~Hz})$, HighBeta $(25-30 \mathrm{~Hz})$ were recorded.

The changes obtained by QEEG in children with ASD in dorsolateral prefrontal cortex (F3-F4) area 46 resulted in normalization of medians of the Z-Score parameter, that were noted, particularly in improvement of higher cognitive functions such as: switching attention, working/short-term memory, perceptual attention associated with consciousness, cognitive flexibility, planning, maintaining abstract rules, and inhibiting inappropriate responses. Similar results were reported by other authors [49-52].

Changes in areas of the brain also were seen in improved functions of such reflexes, as: ATNR, Hands Grasp, STNR, Hands Pulling, and also cognitive Pavlov Orientation.

Electrodes activity recording from central and pre-central areapost-motor cortex: c3 (left) and c4 (right) area of Roland Groove: Main Functions of these areas: planning, control, and execution of voluntary movements, and particularly:

a) The primary motor cortex-control the execution of movement;

b) The premotor cortex (area 4)- the sensory guidance of movement, preparation for movement, spatial guidance of reaching, direct control of proximal and trunk muscles of the body; also mirror neurons-basis of imitating the actions through activation of own motor control circuits;

c) The supplementary motor area-internally generated planning of movement, the planning of sequences of movement, and the coordination of the two sides of the body for bi-manual coordination; muscles, body parts, and both sides of the body control; planning a sequence/repertoire of complex locomotion (e.g., climbing) [46].

The Roland groove electrodes recorded the activity mainly from area of C3 (around the Roland Groove area); and C4 (mid-central electrode)-from central and pre-central areas, which are presented in the figures $3 \mathrm{~A}$ and $3 \mathrm{~B}$.

The data in figures $3 \mathrm{~A}$ and $3 \mathrm{~B}$, and table $3 \mathrm{~A}$ shows the median distribution for individual EEG frequency bands (1-25 Hz), for the C3 electrode (from the left of the Roland fracture area)), before and after MNRI intervention.

A statistically significant reduction in EEG activity for Beta (12-25 $\mathrm{Hz})$, Beta1 $(12-15 \mathrm{~Hz})$, Beta2 $(15-18 \mathrm{~Hz})$ and Beta3 $(18-25 \mathrm{~Hz})$ can explain the noted changes in children with the ASD after the MNRI treatment and, particularly, their more extended ability to: focus, think in terms of cause-effect relations, function on higher consciousness level, be better present for external representative system (perception of wider range of surrounding objects and people), and be more curious and motivated.

The remaining frequency bands did not record any statistically significant differences.

The data from figures $3 \mathrm{C}$ and $3 \mathrm{D}$, and table $3 \mathrm{~B}$, make up the median distribution for individual EEG wave frequency bands $(1-25 \mathrm{~Hz})$, 

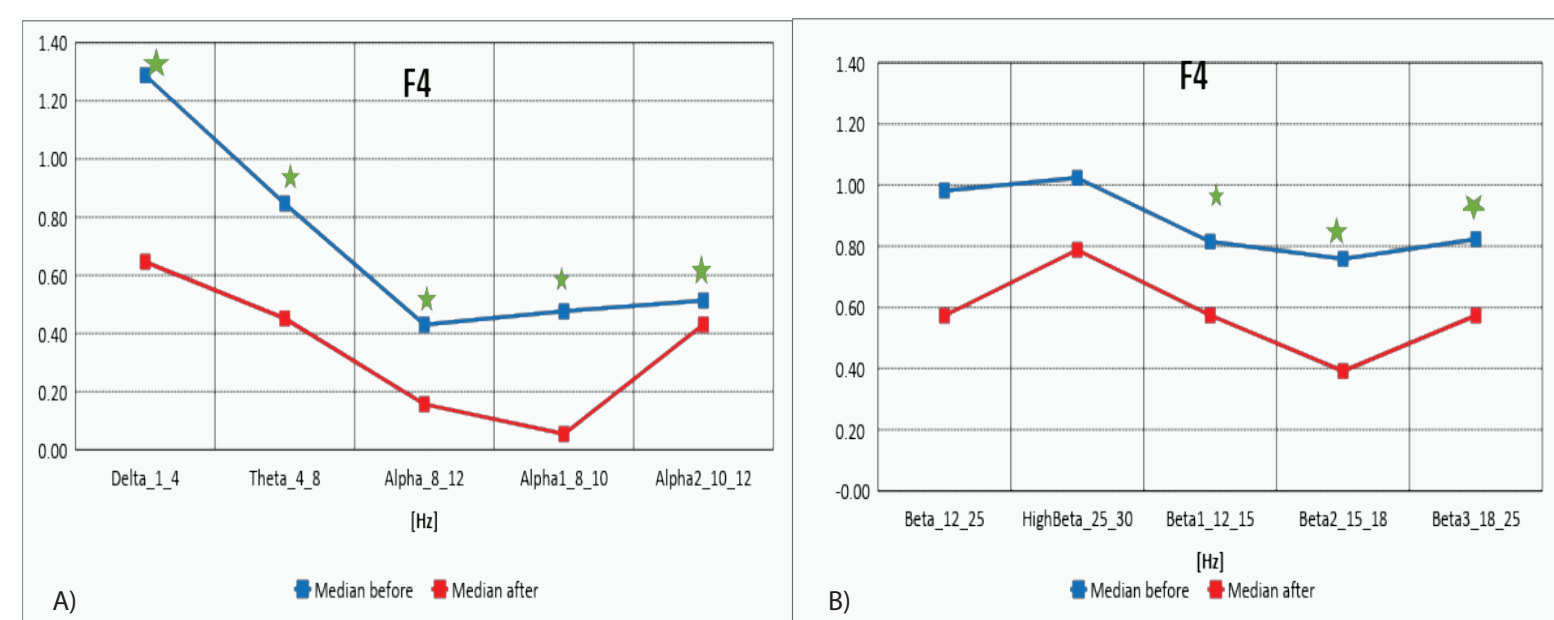

Figure 2A: Median distribution for individual EEG wave frequency bands from 1-12 Hz, for F4 electrode (right area of the brain lobes), before (blue) and after (red) MNRI intervention in children with the ASD aged 6-11y.o. $(n=30)$.

2B): Median distribution for individual EEG wave frequency bands from $12-25 \mathrm{~Hz}$, for F4electrode (right area of the brain frontal lobes), before (blue) and after (red) MNRI intervention in children with the ASD aged 6-11 y.o. ( $n=30)$.

Table 2A: Results of comparative analysis of changes in EEG wave frequency band from 1-25 Hz, for F4electrodebefore and after MNRI intervention in children with the ASD aged6-11y.o. ( $n=30)$. [Legend: in red shows statistical significance].

\begin{tabular}{|c|c|c|c|c|c|c|c|c|c|c|c|c|c|}
\hline F4 & Obs & Median & Mean & Std. Dev. & Min & Max & Obs & Median & Mean & Std. Dev. & Min & Max & $\mathbf{P}$ \\
\hline Delta_1_4_F4 & 30 & 1,287 & 1,414 & 1,264 & $-1,014$ & 4,765 & 27 & 0,647 & 0,844 & 1,363 & $-2,220$ & 3,677 & 0,012 \\
\hline Theta_4_8_F4 & 30 & 0,847 & 0,820 & 0,981 & $-1,635$ & 2,576 & 27 & 0,452 & 0,452 & 1,051 & $-2,408$ & 2,581 & 0,006 \\
\hline Alpha_8_12_F4 & 30 & 0,431 & 0,530 & 0,827 & $-0,816$ & 2,622 & 27 & 0,157 & 0,030 & 0,886 & $-1,915$ & 2,108 & 0,001 \\
\hline Beta_12_25_F4 & 30 & 0,982 & 1,121 & 1,020 & $-0,496$ & 4,056 & 27 & 0,573 & 0,771 & 1,005 & $-0,620$ & 2,815 & 0,007 \\
\hline HighBeta_25_30_F4 & 30 & 1,024 & 1,294 & 1,149 & $-0,380$ & 5,310 & 27 & 0,788 & 0,896 & 1,096 & $-0,565$ & 3,778 & 0,029 \\
\hline Alpha1_8_10_F4 & 30 & 0,476 & 0,445 & 0,900 & $-1,144$ & 2,713 & 27 & 0,055 & 0,004 & 0,846 & $-1,680$ & 2,083 & 0,004 \\
\hline Alpha2_10_12_F4 & 30 & 0,513 & 0,654 & 0,761 & $-0,534$ & 2,274 & 27 & 0,431 & 0,123 & 0,984 & $-2,143$ & 2,042 & 0,002 \\
\hline Beta1_12_15_F4 & 30 & 0,815 & 1,145 & 0,995 & $-0,129$ & 3,453 & 27 & 0,574 & 0,654 & 0,894 & $-0,862$ & 2,316 & 0,007 \\
\hline Beta2_15_18_F4 & 30 & 0,759 & 1,040 & 1,092 & $-0,354$ & 4,298 & 27 & 0,392 & 0,684 & 1,125 & $-0,879$ & 3,377 & 0,006 \\
\hline Beta3_18_25_F4 & 30 & 0,823 & 1,045 & 0,999 & $-0,639$ & 3,823 & 27 & 0,573 & 0,796 & 0,969 & $-0,569$ & 2,798 & 0,027 \\
\hline
\end{tabular}

for the $\mathrm{C} 4$ electrode (to the right of the Roland furrow region in the brain), before and after MNRI intervention. A statistically significant reduction in EEG activity was obtained for Delta (1-4 Hz), High Beta $(25-30 \mathrm{~Hz})$, Beta $(12-25 \mathrm{~Hz})$, Beta2 $(15-18 \mathrm{~Hz})$ and Beta3 $(18-25 \mathrm{~Hz})$. There were no statistically significant differences for the remaining frequency bands.

The normalization of medians of the Z-Score parameter from the central region in areas $\mathrm{C} 3$ and $\mathrm{C} 4$ (and also $\mathrm{Cz}$ ) registered from the sensory-motor cortex involving the voluntary movements, initiation and planning of motor coordination, and also functions of mirror neurons show significant improvements. These functions, usually disturbed in individuals with ASD, affect other areas of their cortex and cerebellum. So this data showing positive changes can be interpreted as the basis for improved balance and posture, and also for improvement in the reflex patterns of: Trunk Extension, STNR, Leg Cross FlexionExtension, Automatic Gait, and Hands Pulling. The cerebellum, as is known, also participates in the modulation of Reticular Formation
(RAS), which stimulates the focusing and attention span coordinated by the cerebral cortex, especially towards new/unknown stimuli [26].

Electrodes activity recording from parietal, angular gyrus (including part of wernicke's area): p4 (right)-Brodmannn area 39: Main functions of these areas: $\mathrm{P} 4$-cognitive processing, auditory decoding, special-temporal info, "Math/Word problem" solving, i.e. reading, writing and interpretation of what is written, non-verbal processing, orienting in three-dimensional space. Lesions to this part of the brain show symptoms of: finger agnosia, alexia (inability to read), acalculia (inability to use arithmetic operations), agraphia (inability to copy), and left-right confusion $[53,54]$.

Mid-frontal electrodes recorded activity mainly from the region of the frontal brain lobes, which is presented on figures $4 \mathrm{~A}$ and $4 \mathrm{~B}$.

The data from figures $4 \mathrm{~A}$ and $4 \mathrm{~B}$, and table 4 show the median distribution for individual EEG wave frequency bands $(1-25 \mathrm{~Hz})$, for the $\mathrm{P} 4$ electrode (from the right parietal region of the brain), before

Citation: Nowak K, Sobaniec P, Sobaniec W, Akhmatova N, Shackleford P (2020) Evaluation of the Therapeutic Effect of MNRI Reflex Neuromodulation on Children Diagnosed with Autism Based on Reflex Assessments, QEEG Analysis and ATEC Questionnaire. J Neurol Neurobiol 6(2): dx.doi.org/10.16966/2379-7150.165 


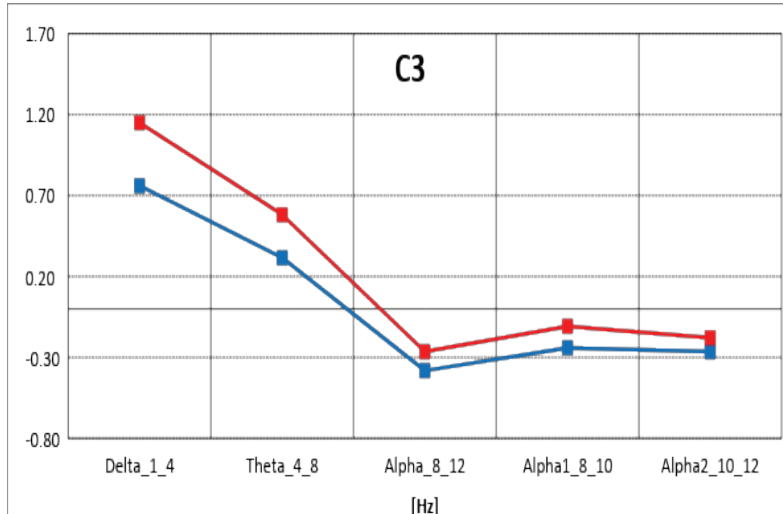

A)

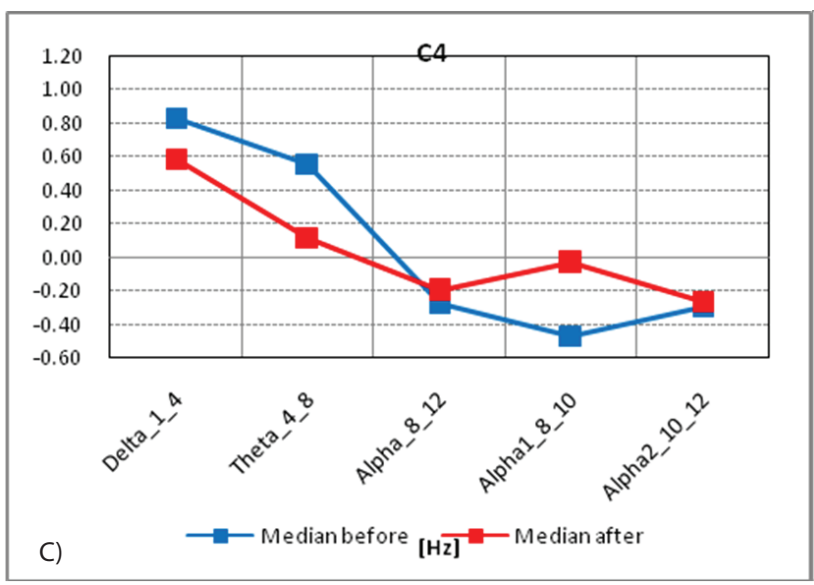

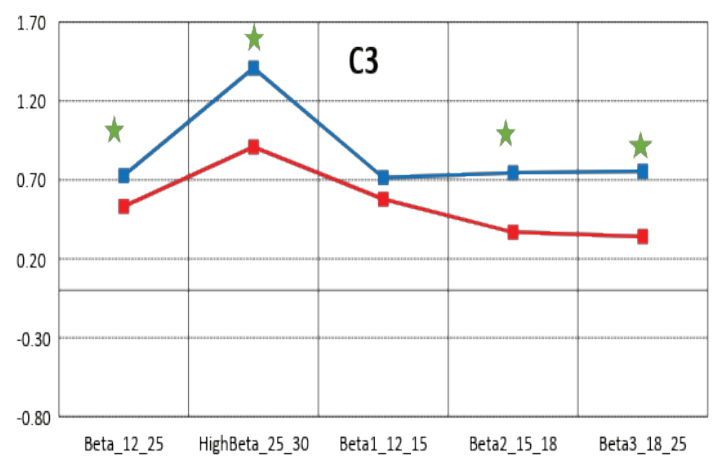

$[\mathrm{Hz}]$

B)

Figure 3A: Median distribution for individual EEG wave frequency bands from 1-12Hz, for C3 electrode (from the left of the Roland fracture area), before (blue) and after (red) MNRI intervention in children with the ASD aged 6-11 ( $n=30)$.

3B): Median distribution for individual EEG wave frequency bands from $12-25 \mathrm{~Hz}$, for C3 electrode (from the left of the Roland fracture area), before (blue) and after (red) MNRI intervention in children with the ASD aged 6-11 $(n=30)$.

3C): Median distribution for individual EEG wave frequency bands from 1-12 Hz, for C4 electrode (from the right of the Roland fracture area), before (blue) and after (red) MNRI intervention in children with the ASD aged 6-11 $(n=30)$.

3D): Median distribution for individual EEG wave frequency bands from $12-25 \mathrm{~Hz}$, for C4 electrode (from the right of the Roland fracture area), before (blue) and after (red) MNRI intervention in children with the ASD aged 6-11 ( $n=30)$.

Table 3A: Results of comparative analysis of changes in EEG wave frequency band results from 1-25 Hz, for C3 electrode before and after MNRI intervention in children with the ASD aged 6-11 $(n=30)$.

\begin{tabular}{|c|c|c|c|c|c|c|c|c|c|c|c|c|c|}
\hline C3 & Obs & Median & Mean & Std. Dev. & Min & Max & Obs & Median & Mean & Std. Dev. & Min & Max & p \\
\hline Delta_1_4_C3-CSD & 30 & 0,760 & 1,226 & 1,502 & $-1,583$ & 6,117 & 27 & 1,149 & 0,842 & 1,073 & $-1,131$ & 2,675 & 0,058 \\
\hline Theta_4_8_C3-CSD & 30 & 0,315 & 0,459 & 1,220 & $-2,209$ & 3,905 & 27 & 0,581 & 0,317 & 1,040 & $-2,503$ & 2,519 & 0,414 \\
\hline Alpha_8_12_C3-CSD & 30 & $-0,381$ & $-0,177$ & 0,717 & $-1,198$ & 1,631 & 27 & $-0,264$ & $-0,160$ & 0,787 & $-1,494$ & 1,376 & 0,923 \\
\hline Beta_12_25_C3-CSD & 30 & 0,727 & 0,723 & 0,938 & $-1,247$ & 2,329 & 27 & 0,532 & 0,504 & 0,791 & $-1,123$ & 2,034 & 0,017 \\
\hline HighBeta_25_30_C3-CSD & 30 & 1,408 & 1,300 & 1,258 & $-0,915$ & 3,860 & 27 & 0,908 & 0,882 & 885 & $-0,950$ & 2,453 & 0,013 \\
\hline Alpha1_8_10_C3-CSD & 30 & $-0,240$ & $-0,083$ & 0,757 & $-1,303$ & 2,180 & 27 & $-0,107$ & $-0,011$ & 0,845 & $-1,167$ & 1,808 & 0,962 \\
\hline Alpha2_10_12_C3-CSD & 30 & $-0,265$ & $-0,205$ & 0,676 & $-1,444$ & 1,285 & 27 & $-0,178$ & $-0,254$ & 0,635 & $-1,592$ & 1,405 & 0,792 \\
\hline Beta1_12_15_C3-CSD & 30 & 0,714 & 0,741 & 0,862 & $-1,094$ & 3,019 & 27 & 0,578 & 0,608 & 1,162 & $-1,109$ & 3,787 & 0,156 \\
\hline Beta2_15_18_C3-CSD & 30 & 0,745 & 0,679 & 1,038 & $-1,716$ & 2,763 & 27 & 0,369 & 0,405 & 0,909 & $-1,075$ & 2,140 & 0,035 \\
\hline Beta3_18_25_C3-CSD & 30 & 0,754 & 0,661 & 0,968 & $-1,234$ & 2,291 & 27 & 0,341 & 0,365 & 0,694 & $-1,075$ & 1,727 & 0,019 \\
\hline
\end{tabular}

Citation: Nowak K, Sobaniec P, Sobaniec W, Akhmatova N, Shackleford P (2020) Evaluation of the Therapeutic Effect of MNRI Reflex Neuromodulation on Children Diagnosed with Autism Based on Reflex Assessments, QEEG Analysis and ATEC Questionnaire. J Neurol Neurobiol 6(2): dx.doi.org/10.16966/2379-7150.165 
Table 3B: Results of comparative analysis of changes in EEG wave frequency band from 1-25 $\mathrm{Hz}$, for P4 electrode before and after MNRI intervention in children with the ASD aged 6-11 $(n=30)$

\begin{tabular}{|c|c|c|c|c|c|c|c|c|c|c|c|c|c|}
\hline C4 & Obs & Median & Mean & Std. Dev. & Min & Max & Obs & Median & Mean & Std. Dev. & Min & Max & $\mathbf{p}$ \\
\hline Delta_1_4_C4-CSD & 30 & 0,830 & 1,011 & 1,236 & $-1,004$ & 4,007 & 27 & 0,586 & 0,644 & 1,350 & $-1,940$ & 4,082 & 0,029 \\
\hline Theta_4_8_C4-CSD & 30 & 0,558 & 0,410 & 1,077 & $-2,123$ & 2,417 & 27 & 0,115 & 0,306 & 1,302 & $-3,047$ & 3,280 & 0,113 \\
\hline Alpha_8_12_C4-CSD & 30 & $-0,274$ & $-0,241$ & 0,875 & $-1,985$ & 1,881 & 27 & $-0,200$ & $-0,275$ & 0,809 & $-1,912$ & 0,986 & 0,701 \\
\hline Beta_12_25_C4-CSD & 30 & 0,520 & 0,677 & 0,835 & $-0,440$ & 2,404 & 27 & 0,278 & 0,409 & 0,801 & $-1,335$ & 2,165 & 0,023 \\
\hline HighBeta_25_30_C4-CSD & 30 & 1,016 & 1,280 & 1,057 & $-0,301$ & 3,410 & 27 & 0,597 & 0,843 & 1,025 & $-1,111$ & 2,905 & 0,021 \\
\hline Alpha1_8_10_C4-CSD & 30 & $-0,467$ & $-0,175$ & 0,918 & $-1,656$ & 2,350 & 27 & $-0,033$ & $-0,125$ & 0,887 & $-1,984$ & 1,501 & 0,631 \\
\hline Alpha2_10_12_C4-CSD & 30 & $-0,295$ & $-0,266$ & 0,768 & $-1,981$ & 1,404 & 27 & $-0,263$ & $-0,379$ & 0,636 & $-1,604$ & 0,592 & 0,337 \\
\hline Beta1_12_15_C4-CSD & 30 & 0,425 & 0,553 & 1,028 & $-1,360$ & 3,851 & 27 & 0,313 & 0,438 & 0,847 & $-0,998$ & 2,575 & 0,387 \\
\hline Beta2_15_18_C4-CSD & 30 & 0,344 & 0,533 & 0,879 & $-0,728$ & 2,802 & 27 & 0,217 & 0,340 & 0,839 & $-1,265$ & 2,043 & 0,046 \\
\hline Beta3_18_25_C4-CSD & 30 & 0,602 & 0,701 & 0,749 & $-0,408$ & 2,575 & 27 & 0,247 & 0,371 & 0,882 & $-1,373$ & 1,955 & 0,013 \\
\hline
\end{tabular}
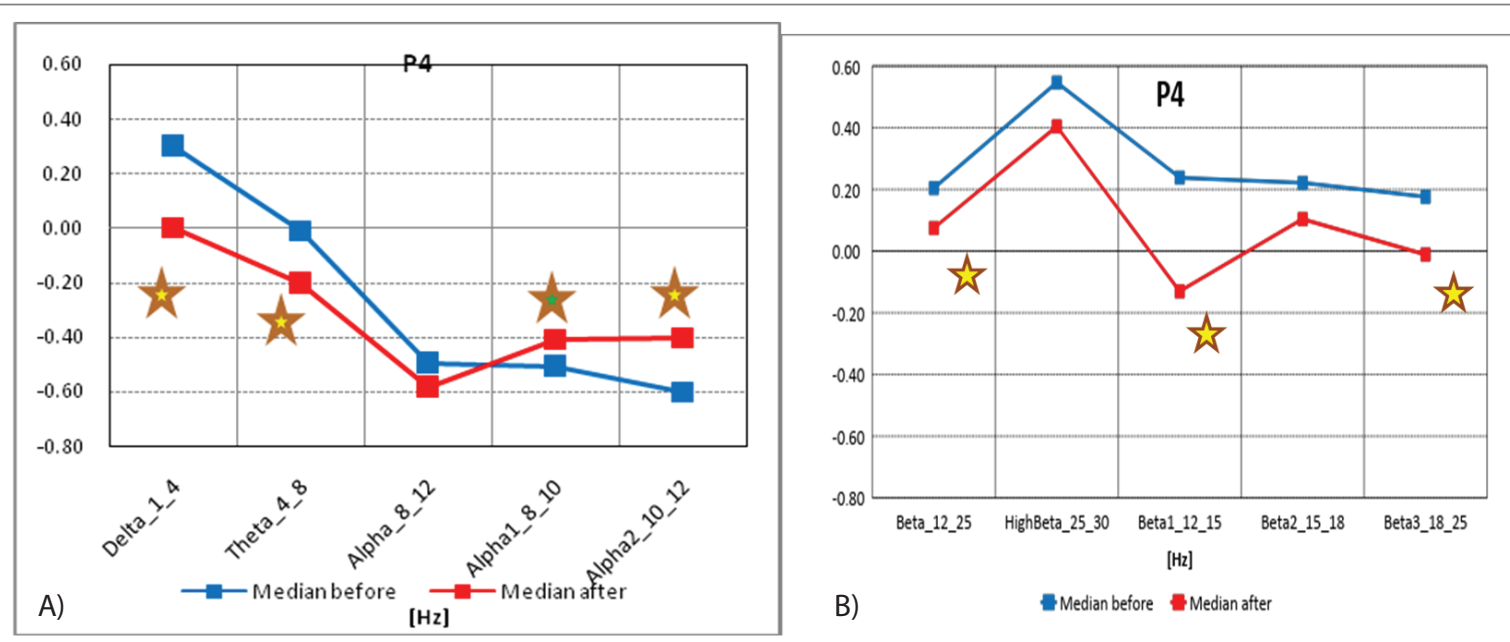

Figure 4A: Median distribution for individual EEG wave frequency bands from 1-12Hz, for P4 electrode (from the right parietal region of the brain), before (blue) and after (red) MNRI intervention in children with the ASD aged 6-11 ( $n=30)$.

4B): Median distribution for individual EEG wave frequency bands from 12-25 Hz, for P4 electrode (right brain region), before (blue) and after (red) MNRI intervention in children with the ASD aged 6-11 $(n=30)$.

and after MNRI intervention. A statistically significant reduction in EEG activity for Alpha2 (10-12 Hz) was obtained. It is important to note that changes in Delta (1-4 Hz), Theta (4-8 Hz), Alpha (10-12 $\mathrm{Hz})$, Betal $(12-15 \mathrm{~Hz})$, and Beta3 $(18-25 \mathrm{~Hz})$ are close to statistical improvements. The remaining frequency bands show changes that are not on significant or close to a significant level.

Analysis of the results of brain mapping in the Wernicke area (P3) prior to and after the MNRI Program in the Study Group shows certain improvements in the electrical potential. These changes were particularly noted as progress in: interests in interaction, ability forn on-verbal communication, attention and memory, and spatialcognitive retrieval, relaxation, managing the inner peace, presence and self-awareness, longer-term focusing, comprehension, reduction of reactivity (startle) for intense audial stimuli.

Electrodes activity recording from dorsolateral prefrontal cortex-p3 (left)-brodmann area $\mathbf{4 6}$ main functions: Area 46 is responsible for saccadic eye movements, emotional expression, encoding, behavioral inhibition (of impulsiveness, calming down, sleeping), calculation, music enjoyment, cognitive processing, special-temporal info, "Math/Word problem" solving, and verbal processing [45].

Mid-frontal electrodes recorded activity mainly from left parietal region, which is presented on figures $5 \mathrm{~A}$ and $5 \mathrm{~B}$. The data from figures $5 \mathrm{~A}$ and $5 \mathrm{~B}$, and Table 5 form the median distribution for individual frequency bands of EEG waves (1-25 Hz), for the P3 electrode (from the left parietal region of the brain), before and after MNRI intervention. Statistically significant reduction of EEG activity for Alpha 2 (10-12 $\mathrm{Hz})$ and Beta3 (18-25 Hz) was found. Some improvement which was close to statistically significant changes in Theta $(4-8 \mathrm{~Hz})$ and Alphal $(8-10 \mathrm{~Hz})$ and also in Betal $(12-15 \mathrm{~Hz})$ took place. There were no statistically significant differences for the remaining frequency bands.

Analysis of the results of brain mapping in the Wernicke area (P3) prior and after the MNRI Program in the Study Group shows some certain improvements in the electrical potential power, on statistically significant and some close to significant levels. These changes were particularly noted in improvements: more interests in interaction, increased ability for expressive language, attention and memory, number processing, spatial-cognitive orientation, self-awareness, 
Table 4: Results of comparative analysis of changes in EEG wave frequency band from 1-25 $\mathrm{Hz}$, for P4 electrode before and after MNRI intervention in children with the ASD aged 6-11 ( $n=30)$.

\begin{tabular}{|c|c|c|c|c|c|c|c|c|c|c|c|c|c|}
\hline P4 & Obs & Median & Mean & Std. Dev. & Min & Max & Obs & Median & Mean & Std. Dev. & Min & Max & p \\
\hline Delta_1_4_P3-CSD & 30 & $-0,223$ & 0,209 & 1,100 & $-1,435$ & 3,753 & 27 & $-0,201$ & 0,031 & 1,230 & $-2,268$ & 3,804 & 0,349 \\
\hline Theta_4_8_P4-CSD & 30 & $-0,008$ & $-0,050$ & 0,896 & $-2,622$ & 1,856 & 27 & $-0,206$ & $-0,059$ & 1,147 & $-1,726$ & 4,006 & 0,885 \\
\hline Alpha_8_12_P4-CSD & 30 & $-0,499$ & $-0,601$ & 0,833 & $-2,292$ & 0,495 & 27 & $-0,585$ & $-0,490$ & 0,921 & $-2,342$ & 1,277 & 0,107 \\
\hline Beta_12_25_P4-CSD & 30 & 0,204 & 0,224 & 1,108 & $-1,613$ & 2,912 & 27 & 0,075 & 0,113 & 1,198 & $-1,863$ & 3,331 & 0,337 \\
\hline HighBeta_25_30_P4-CSD & 30 & 0,547 & 0,799 & 1,399 & $-0,772$ & 5,076 & 27 & 0,404 & 0,548 & 1,315 & $-1,157$ & 4,559 & 0,079 \\
\hline Alpha1_8_10_P4-CSD & 30 & $-0,508$ & $-0,559$ & 0,791 & $-2,160$ & 0,607 & 27 & $-0,407$ & $-0,389$ & 0,902 & $-2,242$ & 1,412 & 0,021 \\
\hline Alpha2_10_12_P4-CSD & 30 & $-0,601$ & $-0,451$ & 0,857 & $-2,037$ & 1,383 & 27 & $-0,401$ & $-0,464$ & 0,880 & $-1,969$ & 1,583 & 0,428 \\
\hline Beta1_12_15_P4-CSD & 30 & 0,238 & 0,177 & 1,141 & $-1,894$ & 3,564 & 27 & $-0,130$ & 0,097 & 1,270 & $-1,814$ & 3,364 & 0,442 \\
\hline Beta2_15_18_P4-CSD & 30 & 0,221 & 0,010 & 1,045 & $-2,651$ & 1,769 & 27 & 0,104 & 0,004 & 1,101 & $-2,089$ & 2,443 & 0,904 \\
\hline Beta3_18_25_P4-CSD & 30 & 0,176 & 0,311 & 1,136 & $-1,754$ & 3,662 & 27 & $-0,011$ & 0,137 & 1,076 & $-1,460$ & 3,461 & 0,164 \\
\hline
\end{tabular}
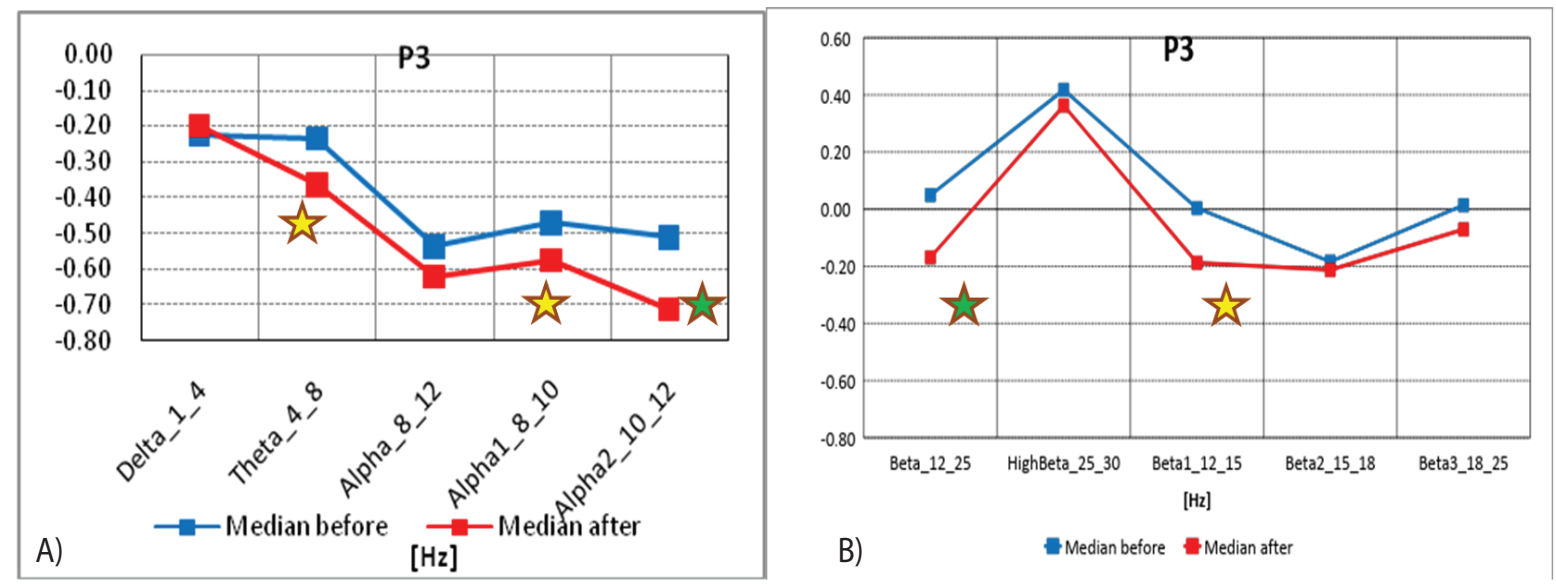

Figure 5A: Median distribution for individual EEG wave frequency bands from 1-12Hz, for P3 electrode (from the left parietal region of the brain), before (blue) and after (red) MNRI intervention in children with the ASD aged 6-11 ( $n=30)$.

5B): Median distribution for individual EEG wave frequency bands from $12-25 \mathrm{~Hz}$, for $\mathrm{P}$ electrode (from the left parietal region of the brain), before (blue) and after (red) MNRI intervention in children with the ASD aged 6-11 $(n=30)$.

Table 5: Results of comparative analysis of changes in EEG wave frequency band from 1-25 Hz, for P3 electrode before and after MNRI intervention in children with the ASD aged 6-11 $(n=30)$.

\begin{tabular}{|c|c|c|c|c|c|c|c|c|c|c|c|c|c|}
\hline P3 & Obs & Median & Mean & Std. Dev. & Min & Max & Obs & Median & Mean & Std. Dev. & Min & Max & $\mathbf{p}$ \\
\hline Delta_1_4_P3-CSD & 30 & $-0,223$ & 0,209 & 1,100 & $-1,435$ & 3,753 & 27 & $-0,201$ & 0,031 & 1,230 & $-2,268$ & 3,804 & 0,349 \\
\hline Theta_4_8_P3-CSD & 30 & $-0,236$ & $-0,041$ & 0,932 & $-1,853$ & 1,588 & 27 & $-0,365$ & $-0,153$ & 1,134 & $-2,364$ & 3,572 & 0,179 \\
\hline Alpha_8_12_P3-CSD & 30 & $-0,537$ & $-0,522$ & 0,856 & $-2,570$ & 1,829 & 27 & $-0,623$ & $-0,664$ & 0,952 & $-2,242$ & 2,089 & 0,773 \\
\hline Beta_12_25_P3-CSD & 30 & 0,049 & 0,193 & 1,016 & $-1,518$ & 2,207 & 27 & $-0,169$ & $-0,068$ & 0,991 & $-1,690$ & 2,675 & 0,075 \\
\hline HighBeta_25_30_P3-CSD & 30 & 0,418 & 0,822 & 1,266 & $-0,716$ & 3,801 & 27 & 0,363 & 0,514 & 1,223 & $-1,191$ & 4,324 & 0,113 \\
\hline Alpha1_8_10_P3-CSD & 30 & $-0,470$ & $-0,504$ & 0,792 & $-2,578$ & 1,710 & 27 & $-0,577$ & $-0,553$ & 0,946 & $-2,216$ & 2,236 & 0,548 \\
\hline Alpha2_10_12_P3-CSD & 30 & $-0,511$ & $-0,391$ & 0,933 & $-2,316$ & 1,704 & 27 & $-0,713$ & $-0,731$ & 0,891 & $-2,140$ & 1,172 & 0,049 \\
\hline Beta1_12_15_P3-CSD & 30 & 0,004 & 0,196 & 1,086 & $-1,641$ & 3,186 & 27 & $-0,188$ & $-0,044$ & 0,978 & $-1,372$ & 2,469 & 0,164 \\
\hline Beta2_15_18_P3-CSD & 30 & $-0,184$ & $-0,013$ & 0,916 & $-1,915$ & 1,841 & 27 & $-0,213$ & $-0,165$ & 0,953 & $-1,837$ & 1,728 & 0,325 \\
\hline Beta3_18_25_P3-CSD & 30 & 0,014 & 0,266 & 0,984 & $-1,155$ & 2,652 & 27 & $-0,071$ & $-0,019$ & 1,058 & $-1,824$ & 3,304 & 0,037 \\
\hline
\end{tabular}


ability for longer-term focusing on fine motor tasks, more complicated sentences in their speech, reduction of auditory reactivity.

Changes in brain areas of P4 and P3 electrodes also were seen in improved functions of such reflexes, as: ATNR, STNR, Stapedial/ Auditory, Babkin Palmomental, Fear Paralysis, Hands Grasp, and also cognitive Pavlov Orientation.

Electrodes activity recording from right orbital, temporal and lateral frontal cortex: frontal eye/visual-f8 (right) area, 45 Brodmann area: Main functions of these areas: Area 45 regulates activity of semantic decision tasks (determining whether a word represents an abstract or a concrete entity), and generation tasks (generating a verb associated with a noun); semantic retrieval or semantic working memory processes; guides recovery of semantic information and evaluates the recovered information.

Right hemisphere F8-lower (or anterior-temporal) electrodes that registered the activity from orbital, temporal and lateral frontal brain region $(\mathrm{F} 8)$ are presented on figures $6 \mathrm{~A}$ and $6 \mathrm{~B}$.

The data from figures $6 \mathrm{~A}$ and $6 \mathrm{~B}$, and table 6 shows the median distribution for individual frequency bands of EEG waves $(1-25 \mathrm{~Hz})$, for the F8 electrode (from the right orbital, temporal and lateral frontal region of the brain), before and after MNRI intervention. Statistically significant reduction of EEG activity for high frequency Beta3 (18$25 \mathrm{~Hz}$ ) was obtained. The changes close to statistically significance were seen for EEG activity for brain waves: Alpha1 (8-10 Hz), High Beta $(25-30 \mathrm{~Hz})$, and Beta2 $(15-18 \mathrm{~Hz})$. There were no statistically significant differences for the remaining frequency bands.

Median decrease and normalization of recording observed in the orbital, temporal and lateral frontal region of cortex (F8) show noted reduction of sensory hypersensitivity, especially to light, frequency of visual system activation and also visual-auditory reactivity resulting in improvement of visual processes: convergence-divergence links, visual focusing, more use of distant vision, visual memory and easier processing of sequencing of objects, ocular-motor, and optokinetic responses. Record of normalization in these areas of their brain maps in these children also resulted in improved abilities for processing of complex visual-auditory objects. Also, this area of the brain known for the executive role of decisions making [21] to positive changes in visual behavior seen in better regulation of distant vision-with less of their typical habit to look from 'too close a distance.' This data correlates with earlier MNRI studies on visual reflexes [39].

Changes in these areas of the brain also were seen in improved functions of such reflexes, as: Hands Grasp, STNR, Hands Pulling, Babkin Palmomental, and Pavlov Orientation.

Electrodes activity recording from central-temporal, temporal and mid-temporal brain region-primary auditory association cortex: T3 (left)/T4 (Right)-Broadman areas 41 and 42: Main functions of these areas: the first cortical destination is the auditory information stemming from the thalamus, neural activity regulation for physical properties of a sound.

T3-T4-Central-temporal electrodes recorded activities are presented on figures $7 \mathrm{~A}$ and $7 \mathrm{~B}$.

The data from figures $7 \mathrm{~A}$ and $7 \mathrm{~B}$, and table $7 \mathrm{~A}$ above show the median distribution for individual EEG frequency bands $(1-25 \mathrm{~Hz})$, for the T3 electrode (from the left frontal-temporal and central temporal region of the brain), before and after MNRI intervention. Statistically significant reduction of EEG activity for Delta (1-4 Hz), Theta (4-8 Hz), Alpha (8-12 Hz), Alpha1 (8-10 Hz), Alpha 2 (10-12 $\mathrm{Hz})$, High Beta $(25-30 \mathrm{~Hz})$, Beta1 $(12-15 \mathrm{~Hz})$, Beta2 $(15-18 \mathrm{~Hz})$ and Beta3 $(18-25 \mathrm{~Hz})$.

The data from figures $7 \mathrm{C}$ and $7 \mathrm{D}$, and table $7 \mathrm{~B}$ shows the median distribution for individual EEG frequency bands $(1-25 \mathrm{~Hz})$, for the T4 electrode (from the right anterolateral and central temporal region of the brain), before and after MNRI intervention. A statistically significant reduction in activity for High Beta $(25-30 \mathrm{~Hz})$, Beta $(12-25$ $\mathrm{Hz})$, Beta2 $(15-18 \mathrm{~Hz})$ and Beta3 $(18-25 \mathrm{~Hz})$ was found. The changes close to statistically significance were seen also for EEG activity for brain waves: Alpha1 $(8-10 \mathrm{~Hz})$, High Beta $(25-30 \mathrm{~Hz})$, and Beta2 $(15-18 \mathrm{~Hz})$. There were no statistically significant differences for the remaining frequency bands.
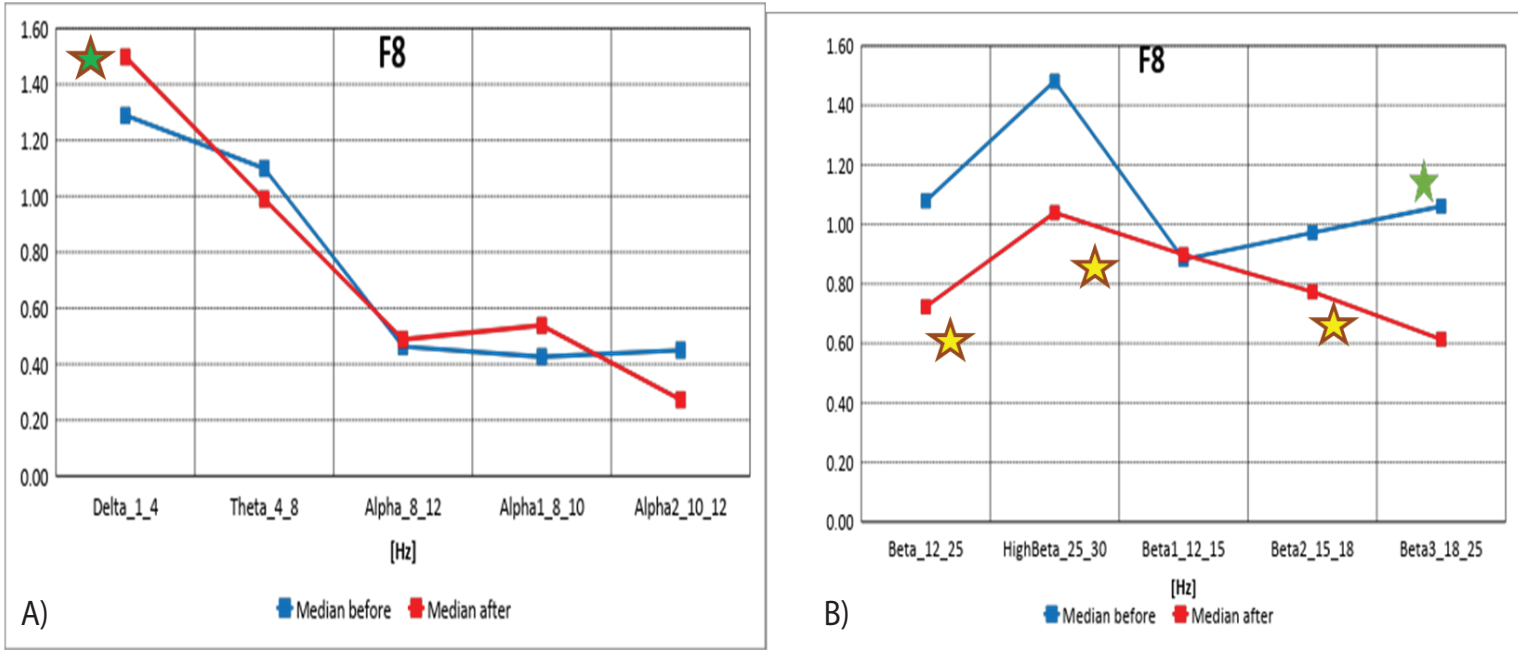

Figure 6A: Median distribution for individual EEG wave frequency bands from 1-12 Hz, for F8. Before (blue) and after (red) MNRI intervention in children with the ASD in age group of 6-11 ( $n=30)$ intervention in children with the ASD aged 6-11 $(n=30)$.

6B): Median distribution for particular EEG wave frequency bands from $12-30 \mathrm{~Hz}$, for $\mathrm{F} 8$ electrode (from the right orbital region, temporal and lateral frontal region of the brain), before (blue) and after (red) MNRI intervention in children with the ASD aged 6-11 ( $n=30)$.

Citation: Nowak K, Sobaniec P, Sobaniec W, Akhmatova N, Shackleford P (2020) Evaluation of the Therapeutic Effect of MNRI Reflex Neuromodulation on Children Diagnosed with Autism Based on Reflex Assessments, QEEG Analysis and ATEC Questionnaire. J Neurol Neurobiol 6(2): dx.doi.org/10.16966/2379-7150.165 
Table 6: Results of comparative analysis of changes in EEG wave frequency band from 1-25 Hz, for P3 electrode before and after MNRI intervention in children with the ASD aged 6-11 $(n=30)$.

\begin{tabular}{|c|c|c|c|c|c|c|c|c|c|c|c|c|c|}
\hline F8 & Obs & Median & Mean & Std. Dev. & Min & Max & Obs & Median & Mean & Std. Dev. & Min & Max & p \\
\hline Delta_1_4_F8-CSD & 30 & 1,289 & 1,791 & 1,586 & $-0,289$ & 4,975 & 27 & 1,498 & 1,679 & 1,603 & $-1,527$ & 5,472 & 0,885 \\
\hline Theta_4_8_F8-CSD & 30 & 1,100 & 1,133 & 0,974 & $-1,189$ & 2,957 & 27 & 0,990 & 1,174 & 1,186 & $-0,641$ & 4,042 & 0,866 \\
\hline Alpha_8_12_F8-CSD & 30 & 0,463 & 0,319 & 0,819 & $-1,227$ & 1,984 & 27 & 0,488 & 0,404 & 0,886 & $-0,988$ & 2,559 & 0,501 \\
\hline Beta_12_25_F8-CSD & 30 & 1,079 & 1,014 & 0,838 & $-0,489$ & 2,551 & 27 & 0,723 & 0,871 & 0,985 & $-1,227$ & 2,503 & 0,230 \\
\hline HighBeta_25_30_F8-CSD & 30 & 1,481 & 1,335 & 0,939 & $-0,218$ & 3,940 & 27 & 1,039 & 1,020 & 0,985 & $-0,833$ & 3,163 & 0,143 \\
\hline Alpha1_8_10_F8-CSD & 30 & 0,427 & 0,250 & 0,890 & $-1,600$ & 2,180 & 27 & 0,539 & 0,371 & 0,886 & $-0,915$ & 2,323 & 0,337 \\
\hline Alpha2_10_12_F8-CSD & 30 & 0,450 & 0,428 & 0,749 & $-1,082$ & 1,621 & 27 & 0,273 & 0,463 & 0,929 & $-0,918$ & 2,933 & 0,701 \\
\hline Beta1_12_15_F8-CSD & 30 & 0,882 & 0,889 & 0,863 & $-0,517$ & 2,666 & 27 & 0,898 & 1,058 & 1,051 & $-0,800$ & 3,199 & 0,387 \\
\hline Beta2_15_18_F8-CSD & 30 & 0,972 & 0,983 & 0,932 & $-1,003$ & 2,819 & 27 & 0,773 & 0,871 & 1,038 & $-1,111$ & 2,737 & 0,517 \\
\hline Beta3_18_25_F8-CSD & 30 & 1,061 & 1,022 & 0,781 & $-0,442$ & 2,841 & 27 & 0,614 & 0,757 & 0,976 & $-1,403$ & 2,515 & 0,021 \\
\hline
\end{tabular}
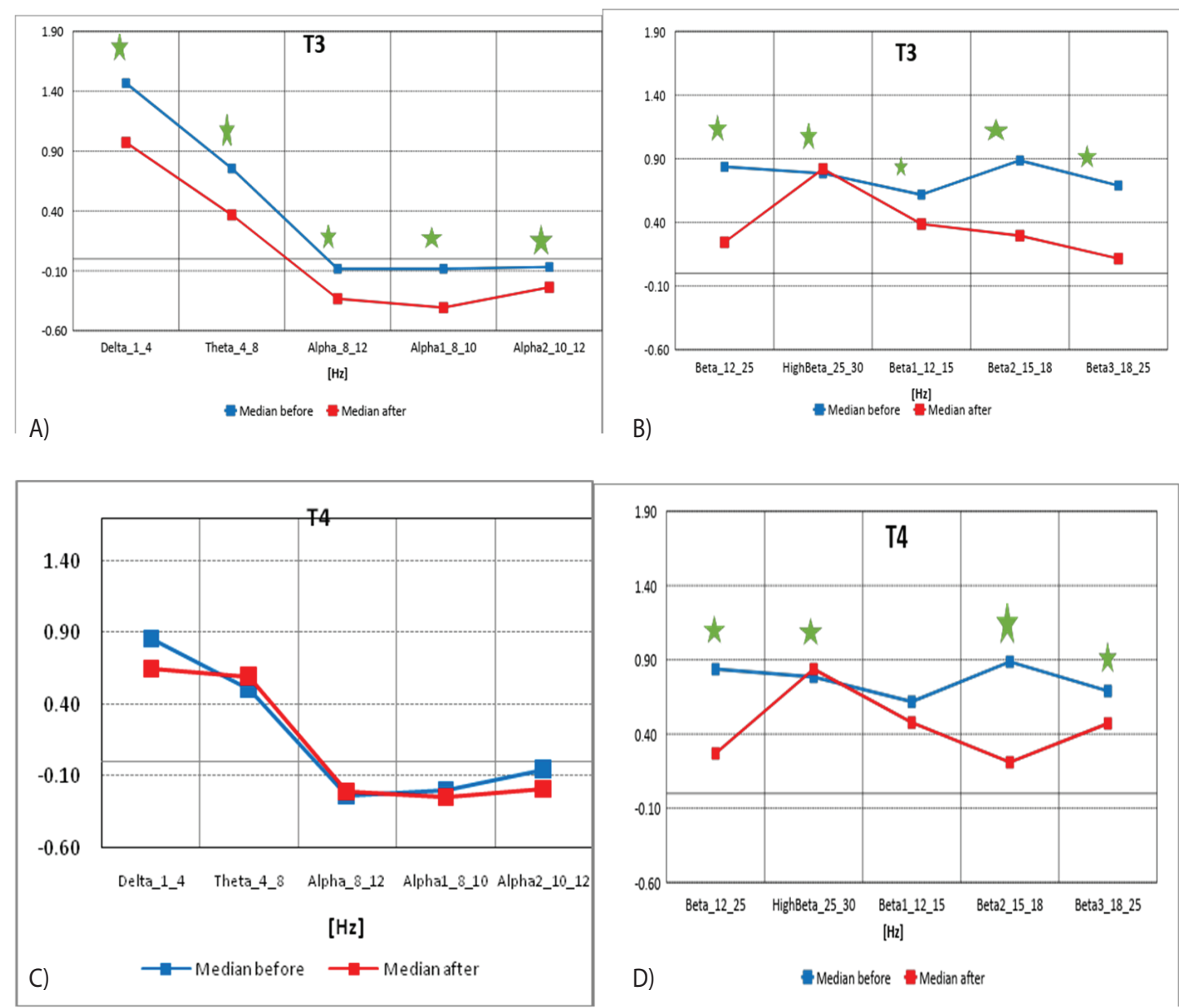

Figure 7A: The median distribution for individual EEG wave frequency bands from $1-12 \mathrm{~Hz}$, for the T3 electrode (from the left frontal-temporal and central temporal region of the brain), before (blue) and after (red) the MNRI intervention in children with the ASD aged 6-11 ( $\mathrm{n}=30$ ).

7B): Median distribution for individual EEG wave frequency range from $12-25 \mathrm{~Hz}$, for T3 electrode (from the left temporo-temporal and central temporal area of the brain), before (blue) and after (red) MNRI intervention in children with the ASD aged 6-11 ( $n=30$ ).

7C): Median distribution for individual EEG wave frequency range from 1-12 Hz, for T4 electrode (from the right frontal-temporal and central temporal area of the brain), before (blue) and after (red) MNRI intervention in children with the ASD aged 6-11 ( $n=30$ ).

7D): Median distribution for individual EEG wave frequency range from 12-25 Hz, for T4 electrode (from the right frontal-temporal and centraltemporal area of the brain), before (blue) and after (red) MNRI therapy intervention in children with the ASD aged 6-11 ( $n=30$ ).

Citation: Nowak K, Sobaniec P, Sobaniec W, Akhmatova N, Shackleford P (2020) Evaluation of the Therapeutic Effect of MNRI Reflex Neuromodulation on Children Diagnosed with Autism Based on Reflex Assessments, QEEG Analysis and ATEC Questionnaire. J Neurol Neurobiol 6(2): dx.doi.org/10.16966/2379-7150.165 
Table 7A: Results of comparative analysis of changes in EEG wave frequency range from 1-25 Hz, for T3 electrode before and after MNRI intervention in children with the ASDaged 6-11 $(n=30)$.

\begin{tabular}{|c|c|c|c|c|c|c|c|c|c|c|c|c|c|}
\hline T3 & Obs & Median & Mean & Std. Dev. & Min & Max & Obs & Median & Mean & Std. Dev. & Min & Max & p \\
\hline Delta_1_4_T3-CSD & 30 & 1,468 & 1,546 & 1,560 & $-1,113$ & 5,470 & 27 & 0,972 & 0,746 & 1,440 & $-2,107$ & 4,018 & 0,001 \\
\hline Theta_4_8_T3-CSD & 30 & 0,755 & 0,609 & 0,849 & $-1,366$ & 2,102 & 27 & 0,367 & 0,314 & 0,964 & $-1,660$ & 3,206 & 0,001 \\
\hline Alpha_8_12_T3-CSD & 30 & $-0,083$ & $-0,013$ & 0,767 & $-1,930$ & 1,382 & 27 & $-0,333$ & $-0,250$ & 0,705 & $-1,896$ & 1,135 & 0,021 \\
\hline Beta_12_25_T3-CSD & 30 & 0,836 & 0,734 & 1,085 & $-1,004$ & 3,038 & 27 & 0,244 & 0,320 & 0,822 & $-1,337$ & 2,004 & 0,004 \\
\hline HighBeta_25_30_T3-CSD & 30 & 0,785 & 1,076 & 1,143 & $-0,684$ & 3,237 & 27 & 0,820 & 0,696 & 0,818 & $-0,793$ & 2,419 & 0,015 \\
\hline Alpha1_8_10_T3-CSD & 30 & $-0,083$ & 0,006 & 0,678 & $-1,395$ & 1,437 & 27 & $-0,408$ & $-0,192$ & 0,681 & $-1,713$ & 1,125 & 0,011 \\
\hline Alpha2_10_12_T3-CSD & 30 & $-0,067$ & 0,070 & 0,816 & $-1,998$ & 2,097 & 27 & $-0,238$ & $-0,186$ & 0,757 & $-1,587$ & 1,839 & 0,033 \\
\hline Beta1_12_15_T3-CSD & 30 & 0,618 & 0,577 & 1,008 & $-1,849$ & 2,855 & 27 & 0,387 & 0,338 & 0,957 & $-1,592$ & 2,825 & 0,044 \\
\hline Beta2_15_18_T3-CSD & 30 & 0,887 & 0,801 & 1,071 & $-1,111$ & 2,886 & 27 & 0,296 & 0,313 & 0,917 & $-1,542$ & 2,136 & 0,001 \\
\hline Beta3_18_25_T3-CSD & 30 & 0,690 & 0,728 & 1,021 & $-0,904$ & 2,603 & 27 & 0,115 & 0,327 & 0,755 & $-0,887$ & 1,895 & 0,005 \\
\hline
\end{tabular}

Table 7B: Results of comparative analysis of changes in EEG wave frequency range from 1-25 Hz, for T4 electrode before and after MNRI intervention in children with the ASD aged 6-11 ( $n=30)$.

\begin{tabular}{|c|c|c|c|c|c|c|c|c|c|c|c|c|c|}
\hline T4 & Obs & Median & Mean & Std. Dev. & Min & Max & Obs & Median & Mean & Std. Dev. & Min & Max & p \\
\hline Delta_1_4_T4-CSD & 30 & 0,862 & 1,089 & 1,022 & $-0,822$ & 3,091 & 27 & 0,653 & 0,775 & 1,436 & $-3,146$ & 3,396 & 0,212 \\
\hline Theta_4_8_T4-CSD & 30 & 0,510 & 0,535 & 0,699 & $-0,661$ & 2,503 & 27 & 0,590 & 0,427 & 1,092 & $-2,765$ & 3,332 & 0,337 \\
\hline Alpha_8_12_T4-CSD & 30 & $-0,232$ & $-0,153$ & 0,550 & $-0,980$ & 1,283 & 27 & $-0,206$ & $-0,245$ & 0,656 & $-1,707$ & 0,970 & 0,548 \\
\hline Beta_12_25_T4-CSD & 30 & 0,724 & 0,768 & 0,672 & $-0,533$ & 2,311 & 27 & 0,269 & 0,339 & 0,835 & $-1,518$ & 2,077 & 0,006 \\
\hline HighBeta_25_30_T4-CSD & 30 & 1,106 & 1,151 & 0,762 & $-0,133$ & 2,422 & 27 & 0,837 & 0,696 & 0,818 & $-1,412$ & 1,817 & 0,008 \\
\hline Alpha1_8_10_T4-CSD & 30 & $-0,192$ & $-0,179$ & 0,536 & $-1,029$ & 1,150 & 27 & $-0,242$ & $-0,181$ & 0,652 & $-1,334$ & 0,988 & 0,701 \\
\hline Alpha2_10_12_T4-CSD & 30 & $-0,056$ & 0,028 & 0,642 & $-0,964$ & 2,219 & 27 & $-0,184$ & $-0,227$ & 0,790 & $-2,211$ & 1,564 & 0,072 \\
\hline Beta1_12_15_T4-CSD & 30 & 0,522 & 0,551 & 0,785 & $-0,631$ & 2,945 & 27 & 0,477 & 0,280 & 0,938 & $-2,033$ & 2,681 & 0,088 \\
\hline Beta2_15_18_T4-CSD & 30 & 0,587 & 0,649 & 0,718 & $-0,385$ & 2,361 & 27 & 0,210 & 0,300 & 0,875 & $-1,418$ & 2,439 & 0,002 \\
\hline Beta3_18_25_T4-CSD & 30 & 0,838 & 0,837 & 0,664 & $-0,442$ & 2,029 & 27 & 0,471 & 0,394 & 0,784 & $-1,251$ & 1,817 & 0,005 \\
\hline
\end{tabular}

Disorders in the Brodmannn fields 41 and 42 cause poor auditory awareness, audial hyper-hyposensitivity and poor auditory processing, problems with speech and language development. Improvements in children with autism in this study were seen in progress with brain activity in the Brodmann fields 41 and 42 are evidently connected with: increase of tolerance for audial stimuli with different frequencies, tones and volume; better processing of information, improvement of pragmatic and conversational language, and comprehension as noted also by professionals and parents. This data is similar to functions of the area 41-42 of the primary auditory association cortex that takes part in listening to different frequencies, tones, harmony, intensity of acoustic patterns and volume [34].

Changes in these areas of the brain were also seen in improved functions of such reflexes as: Auditory Fear Paralysis, ATNR, STNR, Babkin Palmomental, Galant, and cognitive Pavlov Orientation.

Electrodes activity recording from posterior temporal cortex: primary auditory associative-area t6 (right): Main functions of these areas: auditory sensations as result of perception, awareness of sound, ability to react reflexively to sounds (link with subcortical processing), dealing with frequency map (a tonotopic map)-decoding/encoding frequency of sounds, differentiating low frequencies from high, identifying and segregating 'auditory objects', sound source orientation (location of a sound) in space, encoding their 'raw' aspects (frequency, distinction of a sound and its pitch, musical pitchor its echoes [55], and decision making [21].

The data from figures $8 \mathrm{~A}$ and $8 \mathrm{~B}$ and table 8 form the median distribution for individual EEG frequency bands $(1-25 \mathrm{~Hz})$ and for the T6 electrode (from the right posterior temporal region of the brain), before and after MNRI intervention. A statistically significant reduction in EEG activity for High Beta $(25-30 \mathrm{~Hz})$, Beta $(12-25 \mathrm{~Hz})$ and Beta3 $(18-25 \mathrm{~Hz})$ has been demonstrated. The changes, close to being statistically significant, were seen also for EEG activity for brain waves: Delta (1-4 Hz), Theta (4-8 Hz), Alpha (8-12 Hz), Alpha1 $(8-10 \mathrm{~Hz})$, Alpha $2(10-12 \mathrm{~Hz})$. There were no statistically significant differences for the remaining frequency bands.

The median decrease and normalization of recordings observed in children with ASD after the MNRI Program in the temporal region of cortex shows noted improvements in the electrodes registering the waves from temporal region in areas of T3, T4, T6 responsible for reduction of sensory hypersensitivity, especially to sounds, and 

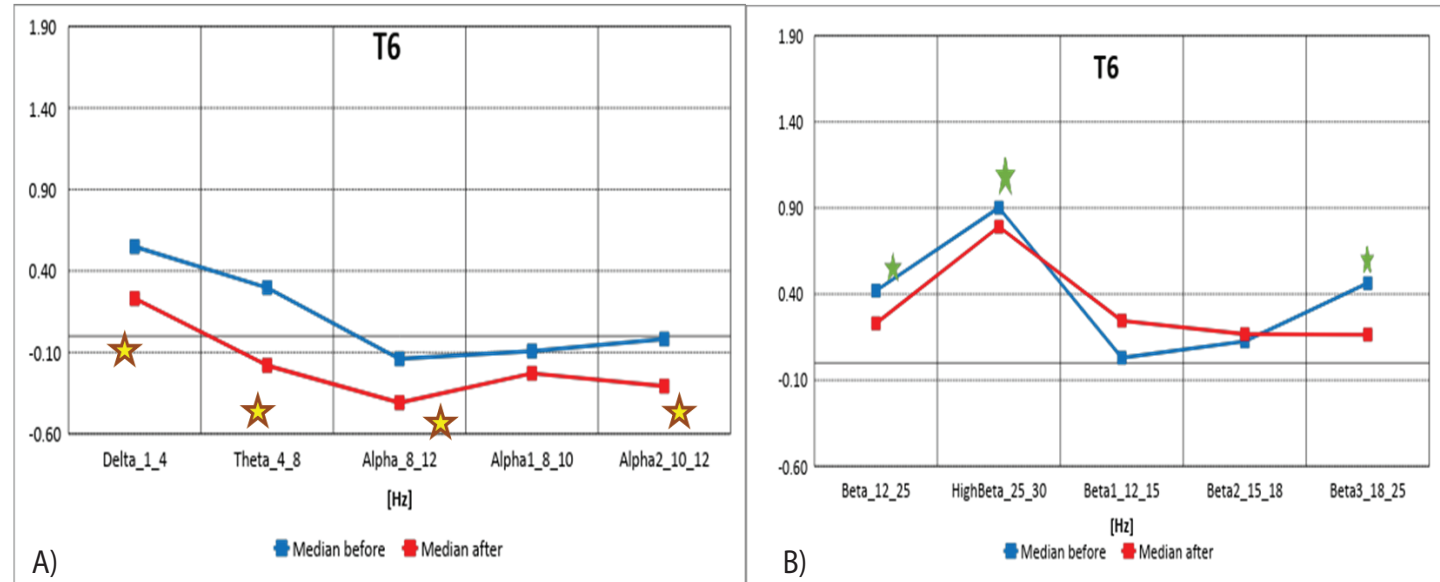

Figure 8A: Median distribution for individual EEG wave frequency range from 1-12 $\mathrm{Hz}$ band, for T6 electrode (from the right temporal region of the brain), before (blue) and after (red) MNRI intervention in children with the ASD aged 6-11 ( $n=30)$.

8B): Median distribution for individual EEG wave frequency range from $12-25 \mathrm{~Hz}$, for T6 electrode (right posterior brain area), before (blue) and after (red) MNRI intervention in children with the ASD aged 6-11 $(n=30)$.

Table 8: Results of comparative analysis of changes in EEG wave frequency range from 1-25 Hz, for T6 electrode before and after MNRI intervention in children with ASD aged 6-11 $(n=30)$.

\begin{tabular}{|c|c|c|c|c|c|c|c|c|c|c|c|c|c|}
\hline T6 & Obs & Median & Mean & Std. Dev. & Min & Max & Obs & Median & Mean & Std. Dev. & Min & Max & $\mathbf{p}$ \\
\hline Delta_1_4_T6-CSD & 30 & 0,549 & 0,631 & 0,886 & $-1,241$ & 3,111 & 27 & 0,230 & 0,194 & 1,241 & $-2,942$ & 3,352 & 0,075 \\
\hline Theta_4_8_T6-CSD & 30 & 0,297 & 0,185 & 0,764 & $-1,369$ & 2,262 & 27 & $-0,180$ & $-0,013$ & 0,986 & $-2,769$ & 2,092 & 0,387 \\
\hline Alpha_8_12_T6-CSD & 30 & $-0,140$ & $-0,239$ & 0,707 & $-1,542$ & 1,199 & 27 & $-0,409$ & $-0,450$ & 0,809 & $-1,884$ & 0,962 & 0,614 \\
\hline Beta_12_25_T6-CSD & 30 & 0,420 & 0,531 & 0,977 & $-1,228$ & 2,544 & 27 & 0,230 & 0,148 & 1,098 & $-2,866$ & 2,053 & 0,026 \\
\hline HighBeta_25_30_T6-CSD & 30 & 0,902 & 1,043 & 0,921 & $-0,609$ & 3,228 & 27 & 0,791 & 0,628 & 1,038 & $-2,053$ & 1,858 & 0,033 \\
\hline Alpha1_8_10_T6-CSD & 30 & $-0,092$ & $-0,243$ & 0,666 & $-1,591$ & 0,963 & 27 & $-0,229$ & $-0,369$ & 0,836 & $-1,975$ & 1,203 & 0,737 \\
\hline Alpha2_10_12_T6-CSD & 30 & $-0,018$ & $-0,097$ & 0,773 & $-1,647$ & 1,987 & 27 & $-0,307$ & $-0,438$ & 0,741 & $-2,463$ & 0,587 & 0,259 \\
\hline Beta1_12_15_T6-CSD & 30 & 0,030 & 0,293 & 0,997 & $-1,400$ & 2,370 & 27 & 0,245 & 0,008 & 1,101 & $-3,260$ & 1,580 & 0,171 \\
\hline Beta2_15_18_T6-CSD & 30 & 0,125 & 0,399 & 1,049 & $-1,264$ & 2,970 & 27 & 0,167 & 0,126 & 1,199 & $-2,719$ & 2,781 & 0,136 \\
\hline Beta3_18_25_T6-CSD & 30 & 0,463 & 0,720 & 0,945 & $-1,003$ & 2,728 & 27 & 0,164 & 0,265 & 1,032 & $-2,103$ & 2,075 & 0,006 \\
\hline
\end{tabular}

indicates improvement in auditory and visual memory, auditory processing, and sequencing of events. Record of normalization in the areas of brain maps in these children also resulted in improved abilities for less reactivity for more intensive/loud sounds, processing of complex sounds, better ability for voice modulation, generating words and sentences. This area of the brain is also known for the executive role of decision making (Nuwer MR. [21]) and showed positive changes in perception of their own behavior.

Changes in this area of the brain also were seen in improved functions of such reflexes, as: ATNR, STNR, Hands Pulling, Babkin Palmomental, Auditory Fear Paralysis, and Pavlov Orientation.

Electrodes activity recording from medial frontal cortex and anterior gyrus: the secondary motor Cortex-Fz Brodmannn area 6: This area is composed of the premotor cortex (a), and, medially, the supplementary motor area (b). Main functions of these areas:

- The premotor cortex-control of the trunk muscles of the body, planning of complex, coordinated movements, the sensory and spatial guidance of movement, direct control of behavior, understanding the actions of others, using abstract rules to perform specific tasks [56].

- The supplementary motor area-contributes to the direct control of movements that are internally generated rather than triggered by sensory events, control of sequential and non-sequential movements (more of temporal character), bilateral and all four limb movements, unimanual and bimanual coordination (stimulus-cued), postural stability while standing or walking, coordinating temporal sequences of actions. This brain area does not play an exclusive role for all these functions, but works on a level of sub coordinating actions and their sequences $[45,47,55,57]$.

Data from figures $9 \mathrm{~A}$ and $9 \mathrm{~B}$ and table 9 show the median distribution for individual EEG wave frequency bands $(1-25 \mathrm{~Hz})$, for the $\mathrm{Fz}$ electrode (from the medial and medial frontal region of the brain), before and after MNRI intervention. A statistically significant reduction in EEG activity for Delta (1-4 Hz), Theta (4-8 $\mathrm{Hz})$, Alpha1 $(8-10 \mathrm{~Hz})$, Beta2 $(15-18 \mathrm{~Hz})$ and Beta3 $(18-25 \mathrm{~Hz})$ has been demonstrated. The changes close to statistical significance were 

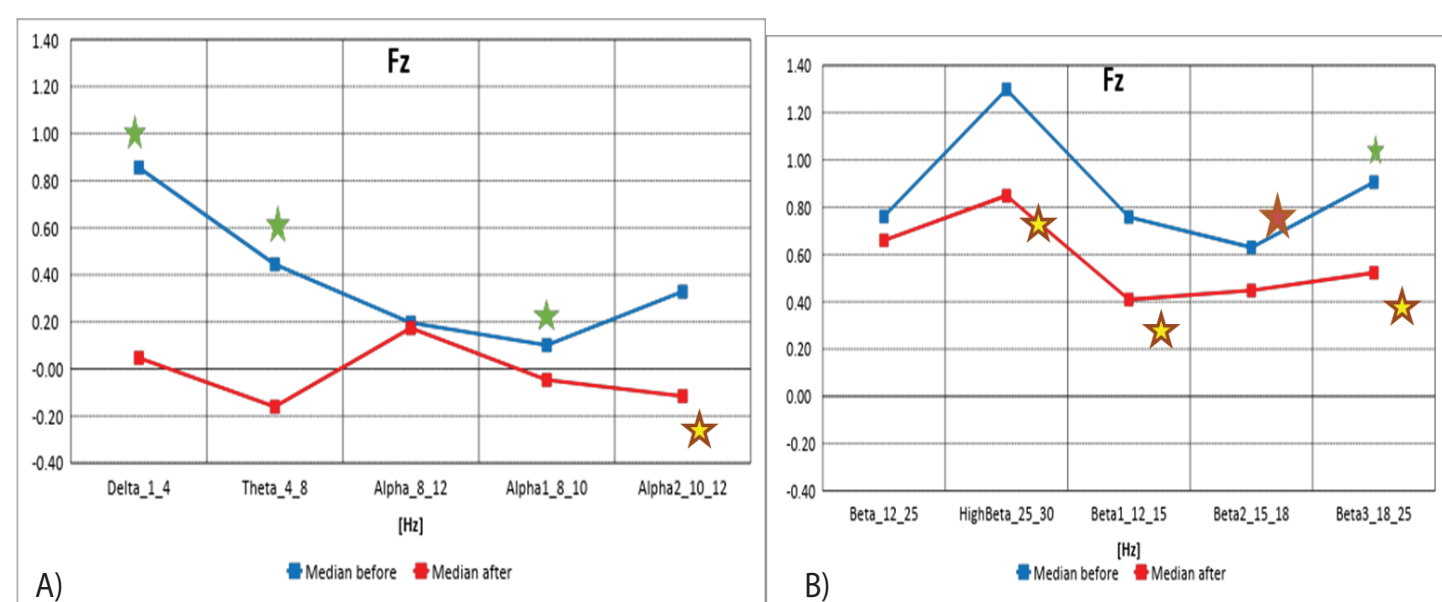

Figure 9A: The median distribution for individual EEG wave frequency range from $1-12 \mathrm{~Hz}$, for the Fz electrode (from the medial and medial frontal region of the brain), before (blue) and after (red) the MNRI intervention in children with the ASD aged 6-11 ( $n=30)$.

9B): Median distribution for individual EEG wave frequency range from $12-25 \mathrm{~Hz}$, for the Fz electrode (from the medial and medial frontal region of the brain), before (blue) and after (red) the MNRI intervention in children with the ASD aged 6-11 $(n=30)$.

Table 9: Results of comparative analysis of changes in EEG wave frequency ranges from 1-25 Hz, for the Fz electrode before and after the MNRI intervention in children with the ASD aged 6-11 $(n=30)$.

\begin{tabular}{|c|c|c|c|c|c|c|c|c|c|c|c|c|c|}
\hline Fz & Obs & Median & Mean & Std. Dev. & Min & Max & Obs & Median & Mean & Std. Dev. & Min & Max & p \\
\hline Delta_1_4_Fz-CSD & 30 & 0,856 & 0,898 & 1,237 & $-0,852$ & 3,672 & 27 & 0,047 & 0,240 & 1,132 & $-1,860$ & 2,081 & 0,010 \\
\hline Theta_4_8_Fz-CSD & 30 & 0,445 & 0,391 & 0,809 & $-0,938$ & 1,653 & 27 & $-0,160$ & 0,107 & 0,982 & $-1,913$ & 1,961 & 0,006 \\
\hline Alpha_8_12_Fz-CSD & 30 & 0,196 & 0,281 & 0,694 & $-0,812$ & 2,195 & 27 & 0,173 & 0,099 & 0,730 & $-1,321$ & 1,569 & 0,239 \\
\hline Beta_12_25_Fz-CSD & 30 & 0,761 & 0,935 & 0,706 & $-0,041$ & 2,684 & 27 & 0,660 & 0,783 & 1,159 & $-1,147$ & 3,697 & 0,179 \\
\hline HighBeta_25_30_Fz-CSD & 30 & 1,299 & 1,433 & 1,126 & 0,131 & 4,666 & 27 & 0,849 & 1,073 & 1,180 & $-1,174$ & 3,179 & 0,203 \\
\hline Alpha1_8_10_Fz-CSD & 30 & 0,101 & 0,204 & 0,789 & $-0,994$ & 2,472 & 27 & $-0,046$ & $-0,050$ & 0,763 & $-1,430$ & 1,654 & 0,041 \\
\hline Alpha2_10_12_Fz-CSD & 30 & 0,328 & 0,349 & 0,580 & $-0,810$ & 1,567 & 27 & $-0,115$ & 0,274 & 0,894 & $-1,040$ & 1,831 & 0,442 \\
\hline Beta1_12_15_Fz-CSD & 30 & 0,759 & 0,910 & 0,958 & $-0,534$ & 3,115 & 27 & 0,410 & 0,884 & 1,609 & $-1,040$ & 5,241 & 0,456 \\
\hline Beta2_15_18_Fz-CSD & 30 & 0,630 & 0,743 & 0,787 & $-0,571$ & 2,518 & 27 & 0,448 & 0,432 & 0,845 & $-1,200$ & 2,433 & 0,020 \\
\hline Beta3_18_25_Fz-CSD & 30 & 0,907 & 0,923 & 0,769 & $-0,155$ & 3,236 & 27 & 0,523 & 0,636 & 0,844 & $-1,270$ & 2,176 & 0,044 \\
\hline
\end{tabular}

seen for EEG activity for brain waves: Alpha2 (10-12 Hz), High Beta $(25-30 \mathrm{~Hz})$, Beta1 $(12-25 \mathrm{~Hz})$, and Beta3 $(18-25 \mathrm{~Hz})$. There were no statistically significant differences for the remaining frequency bands.

The Fz electrode-Brodmannn field 6 [56]-recording the frequencies from this area of the secondary motor cortex and indirectly the anterior gyrus (deep brain structures) known for the initiation of proximal muscles and the torso. The left sensorymotor region participates in maintaining speech, thus damage to this area can cause kinetic apraxia [53]. Studies with the use fMRI have shown that in individuals with ASD, structural and functional ASD disorders have the tendency for rigid and stereotypical behavior and fear of new situations [53].

This study shows significant improvements in children with the ASD after the MNRI Program, and particularly noted in the reduction of improper activity in fast brain wave frequencies-Beta2, Beta3, and Alphal causing the tendency for motor over-reactivity and discoordination; and also the reduction of improper slow frequenciesDelta and Theta-meaning immaturity of brain functions [58].

Normalization in these areas of brain maps implies improved abilities for less reactivity in spontaneous movements, better control of motor coordination systems and posture. Also, this is the area of the brain known for the executive role of motor planning of complex coordinated movements [54] and, as such, showed positive changes in children with ASD as seen in improvements of: motor coordination and control, motor-cognitive behavior and ability to focus on physical hands-on tasks and accomplishment of instructions.

Changes in these areas of the brain were seen in improved functions of such reflexes, as: Leg Cross Flexion-Extension, Automatic Gate, Trunk Extension, STNR, Hands Pulling, and kinesthetic aspect of Pavlov Orientation. 

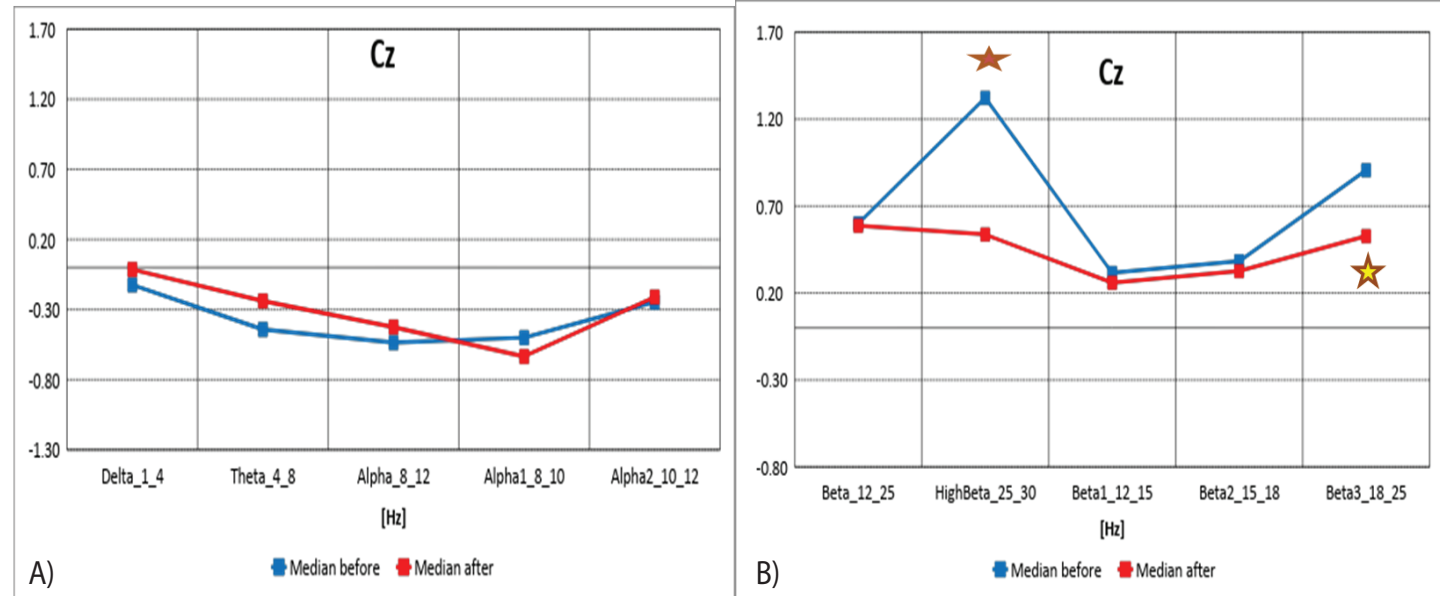

Figure 10A): Median distribution for individual EEG wave frequency range from 1-12 Hz, for the $\mathrm{Cz}$ (central and medial central region of the brain) before (blue) and after (red) MNRI intervention in children with the ASD aged 6-11 $(n=30)$.

10B): Cumulative comparison of relative changes in EEG wave band frequency ranges from 1-25 Hz, for $\mathrm{Cz}$ electrode before and after MNRI intervention in children with the ASD aged6-11 ( $n=30)$.

Table 10: Cumulative comparison of relative changes in EEG wave band frequency ranges from 1-25 Hz, for $\mathrm{Cz}$ electrode before and after MNRI intervention in children with the ASD aged6-11 $(n=30)$.

\begin{tabular}{|c|c|c|c|c|c|c|c|c|c|c|c|c|c|}
\hline $\mathbf{C z}$ & Obs & Median & Mean & Std. Dev. & Min & Max & Obs & Median & Mean & Std. Dev. & Min & Max & p \\
\hline Delta_1_4_Cz-CSD & 30 & $-0,124$ & 0,280 & 1,257 & $-1,592$ & 2,831 & 27 & $-0,015$ & 0,002 & 1,455 & $-3,315$ & 3,337 & 0,072 \\
\hline Theta_4_8_Cz-CSD & 30 & $-0,442$ & $-0,098$ & 1,136 & $-2,045$ & 2,859 & 27 & $-0,239$ & $-0,039$ & 1,425 & $-3,031$ & 3,953 & 0,923 \\
\hline Alpha_8_12_Cz-CSD & 30 & $-0,536$ & $-0,255$ & 1,035 & $-1,872$ & 1,670 & 27 & $-0,425$ & $-0,314$ & 1,093 & $-2,394$ & 1,997 & 0,631 \\
\hline Beta_12_25_Cz-CSD & 30 & 0,601 & 0,670 & 1,014 & $-0,853$ & 4,165 & 27 & 0,587 & 0,369 & 0,922 & $-1,288$ & 2,393 & 0,079 \\
\hline HighBeta_25_30_Cz-CSD & 30 & 1,322 & 1,213 & 1,039 & $-1,075$ & 3,917 & 27 & 0,537 & 0,583 & 0,860 & $-0,780$ & 2,134 & 0,0003 \\
\hline Alpha1_8_10_Cz-CSD & 30 & $-0,500$ & $-0,265$ & 1,090 & $-1,924$ & 1,784 & 27 & $-0,634$ & $-0,302$ & 1,071 & $-2,102$ & 2,067 & 0,719 \\
\hline Alpha2_10_12_Cz-CSD & 30 & $-0,246$ & $-0,180$ & 0,823 & $-1,336$ & 1,787 & 27 & $-0,211$ & $-0,302$ & 1,030 & $-2,438$ & 1,338 & 0,259 \\
\hline Beta1_12_15_Cz-CSD & 30 & 0,316 & 0,527 & 1,287 & $-1,003$ & 5,701 & 27 & 0,259 & 0,248 & 1,087 & $-1,574$ & 2,467 & 0,079 \\
\hline Beta2_15_18_Cz-CSD & 30 & 0,383 & 0,479 & 0,894 & $-1,028$ & 2,946 & 27 & 0,326 & 0,280 & 0,952 & $-1,272$ & 2,361 & 0,088 \\
\hline Beta3_18_25_Cz-CSD & 30 & 0,906 & 0,713 & 0,788 & $-0,734$ & 2,143 & 27 & 0,527 & 0,423 & 0,872 & $-1,100$ & 2,240 & 0,016 \\
\hline
\end{tabular}

Electrodes activity recording from medial central and precentral cortex: somato-sensory association (pre-central gyrus)-cz area: The electrode recording the electrical potential from this brain area are: Brodmann fields 4 (precentral gyrus or primary motor area), 6 (premotor or supplemental motor area) and 1, 2, 3 (primary sensory strip area). Cell bodies of the pyramidal tract are located in this part of the brain.

Main Functions of these areas: decoding/encoding of tactile and proprioceptive sensations, and kinesthesia, movements of skeletal muscles, planning (also for the future), complex behaviors conducting (predicting) the consequences of one's actions, impulse control, contributes to personality development, focusing attention, anticipating events in the environment, visual recognition, emotional control (empathy, shame, compassion and guilt), social interactions, and motivation/reward, short-term memory tasks [20,47]. Damage to this part of the brain diminishes the capacity for sensory-motor processing and social emotions (though leaves logical reasoning intact) [46].
Data from figures $10 \mathrm{~A}$ and $10 \mathrm{~B}$, and table 10 show the median distribution for individual EEG wave frequency bands $(1-25 \mathrm{~Hz})$ for the $\mathrm{Cz}$ (central and medial central region of the brain) before and after MNRI intervention. A statistically significant reduction in EEG activity for High Beta $(25-30 \mathrm{~Hz})$ has been demonstrated. Changes close to being statistically significant were seen for Beta3 $(18-25 \mathrm{~Hz})$. There were no statistically significant differences for the remaining frequency bands.

In our research, normalization of medians of the Z-Score parameter from the central region of the brain in the children with ASD, visible in the areas $\mathrm{Cz}$ (also C3, C4-see above) located in the area of the sensory-motor cortex $[25,52]$ noted improvements in sensory-motor integration, decrease of hypersensitivity on lower limbs, normalization of sensation in upper limbs, improvement in voluntary movements and actions $v s$. motor and emotional reactivity, maintaining balance and correct posture, better self-awareness and presence, also a tendency for observing the actions of others and imitating their movements and the manners of their interaction.

Citation: Nowak K, Sobaniec P, Sobaniec W, Akhmatova N, Shackleford P (2020) Evaluation of the Therapeutic Effect of MNRI Reflex Neuromodulation on Children Diagnosed with Autism Based on Reflex Assessments, QEEG Analysis and ATEC Questionnaire. J Neurol Neurobiol 6(2): dx.doi.org/10.16966/2379-7150.165 
Changes in these areas of the brain also were seen in improved functions of such reflexes, as: Leg Cross Flexion-Extension, Automatic Gate, Trunk Extension, STNR, Hands Pulling, and kinesthetic aspect of Pavlov Orientation.

Table 2 Results of comparative analysis of changes in EEG wave frequency band from $1-25 \mathrm{~Hz}$, for F4 electrode before and after MNRI intervention in children with the ASD aged 6-11y.o. $(n=30)$. [Legend: in red shows statistical significance].

\section{ATEC Evaluation Test Results and Discussion of Progress of Children with the MNRI Program}

The results of the ATEC test aimed at measuring the common (categories) and particular symptoms (features) of autism in children seen in social deficits, communication impairment, and rigid repetitive or stereotyped behavior prior and after the MNRI therapy are presented in figures below [Figures 11-14].

\section{ATEC results-category 1: speech, language, and communication}

Parents of $31 \%$ of children noted changes (statistically significant level) of $31 \%$-close to statistical significance. The main achievements noted by parents were: greater changes and increase in abilities for non-verbal and verbal communication, better focusing, responding properly to their own names, and to the word "No."

Analysis of questions in the ATEC test category I-Speech, language, communication, demonstrated some statistically significant changes in the ATEC evaluation [Figure11] after the 8-day MNRI Program.

The significant changes are noted in abilities for "talking on a rather good level corresponding to their age" (1) "started using several sentences in their speech and communication" (2), "functional speech reflects the content and corresponds the situation" (3), "asks meaningful and reasonable questions" (4), and "can ask what he/she wants" (5). All these improved qualities are of a high importance in real life situations and usually needs months and years of training before showing the perspective improvements in children with ASD with the use of the MNRI intervention tools.

Clinical research and observations of specialists and parents also showed positive changes in other features of this category, beyond that which are presented in the ATEC Questionnaire, specifically, important changes in precursors for speech/language/communication development as seen in a much higher level of desire to communicate (initiation of interaction/s), changes in voice modulation; nonverbal children were showing the elements of imitation of speech increasing the spectrum of syllables and words, ability for repetition, spontaneous syllables/words/sentences pronunciation, better focusing on instructions, bettering their state of 'presence' vs. 'absence', and more patience and cooperation. These changes increased after the Conference/s and moved on to a significant level after the next 1-3 months according to the parents' and local therapist reports.

\section{ATEC-Result on category 2: socialization}

The results of the ATEC questionnaire for the category 2-Socialization after the MNRI intervention showed a significant improvement for answers 2.10, 2.11, 2.12, 2.15, which concern interpersonal interactions and behavior [Figure 12].

Observation also show additionally positive changes in other socializing features beyond that presented in the ATEC Questionnaire particularly: improvements in more desire to interact with people around them, willingness to be around others for a longer time, watching others and interest in their actions and behavior, more patience and inner peace while communicating, the ability to ask for help in a clearer way, and better comprehension of links between surrounding people.

Overall, parents of $48 \%$ of children noted changes (statistically significant level) of $22 \%$-close to statistical significance. The main achievement in this category is evidenced in greater reduction of tantrums in all participants, increasing imitation abilities, the desire to hug, and sharing property.

\section{ATEC Results-Category3: Sensory/Cognitive Awareness}

Significant improvements in category 3-Sensory/Cognitive Awareness are noted for points 3.2, 3.6, 3.7, which are characterized by a proper reaction to the environment and the skillful use of toys from the immediate environment [Figure 13].

Observation showed positive changes in other sensory and somato sensory exploration markers beyond those measured in the ATEC Questionnaire particularly in: improvement in focusing for vision and listening, decrease of sensitivity of touch to skin (permission for touch and hugs for parents and close people), improved regulation of sensitivity of palms, neck/face/head, feet, legs and trunk areas. Other areas that these observations were noted included improvements in interest for texture and other objects, interest in learning own body parts including naming them.

Overall, parents of $38 \%$ of participants noted changes (statistically significant level), of $16 \%$-close to statistical significance. The main achievement in this Category shows greater changes in response from being praised, appropriate use of toys, proper emotional and facial expressions.

\section{ATEC results-category 4: health/physical/behavior}

Analysis of changes in the Category 4 "Physical Health, Behavior" shows a significant improvement in questions: 4.5, 4.6, 4.7, 4.9, 4.13, $4.14,4.15,4.16,4.19,4.20,4.22,4.23,4.24,4.25$. The common feature is calming down, presence, silencing, decrease of hypersensitivity, agitation, stereotypical behavior, aggression, auto-aggression [Figure 14]. Observation showed additionally positive changes in other physical markers beyond that measured in the ATEC Questionnaire: improvement in the speed of movements, more flexibility, more mobility and coordination in body plane movements, desire to repeat more complicated movements evidencing the increased ability for motor programming and control; also less toe walking and more normal walking (stepping on heels and whole feet before shifting the body weight to toes), better postural control and freedom for movements of the horizontal plane to explore the surrounding are noted. The use of more distant vision vs. looking at objects within a shortened distance increased significantly.

Overall, parents of $59 \%$ of participants noted changes (statistically significant level), of $10 \%$-close to statistical significance. They noted improvement in sleeping patterns, reduction of auditory sensitivity, improvement in psychomotor silence/relaxation, reduction of frustration and agitation, as well as, release of rigid patterns and behaviors, the decrease of tactile and sonic sensitivity, improvement in the work of the gastrointestinal tract: less constipation and more normalization in eating.

\section{Discussion}

Individuals with global developmental disorders such as ASD demonstrate a very extensive range of clinical symptoms and challenges [59] such as: deficits in communication and social interaction; limited 


\section{ATEC test \\ Speech, language, communication, $\mathrm{N}=30$}

1.13. Carries on fairly good conversation

1.11. Speech tends to be...
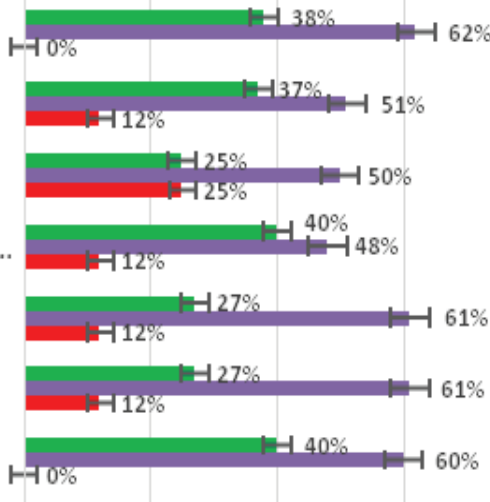

1.10. Asksmeaningful

1.9. Explains what he/she wants

1.7. Knows 10 or more words

1.3. Can follow some commands

1.2. Responds to 'No' or 'Stop'

1.1 Knows own name

$$
-20 \%
$$

$$
0 \%
$$

\% $20 \%$

$40 \%$

$60 \%$

$80 \%$

1 improvement

0 no changes

-1 deterioration

Figure 11: ATEC Questionnaire results: relative distribution of changes in Speech, language, communication category 1 for 30 individuals aged 6-11 years before and after the MNRI therapy.

behavioral patterns, activities and interests occurring in the form of stereotypical movement and/or verbal patterns and atypical sensory behavior, sensory processing disorders, sensory motor hypersensitivity or under sensitivity [9], and excessive attachment to routine and ritualized behavior patterns; also selective, limited interests.

Our research was aimed at improvement of these symptoms by the use of the therapeutic MNRI method. MNRI Neurosensomotor Reflex Integration is a holistic program, using activation of reflex receptive fields and the sense of proprioception mechanisms, sensorimotor integration, and primary sensory-motor responses [40].

MNRI therapy affects the regulatory mechanisms of reflex functions, their induction on appropriate sensory-motor schemes $[19,42,40]$.

L Vygotsky [60] report that primary sensory-motor system (the extra pypamidal part of the nervous system) is the basis for the development of intentional movements that helps in cognitive processing, choice making and achieving goals (pyramidal).

\section{Progress in reflex patterns}

The results of the MNRI Program show remarkable progress in whole range of reflex patterns in children with ASD aged 6-11 years and particularly in $33.33 \% / 10$ out of 30 patterns on highly statistical level. For example, the Core Tendon Guard prior and after the MNRI changed significantly-the average 12.97 points improved up to 13.53 points $(\mathrm{p}=0.0001$ ), noted in improvement in the symmetry of the torso, postural and head righting control, reduction of sensory hypersensitivity within the torso area. Another reflex pattern-Robinson Hands Grasp that was on level of 10.25 points prior the MNRI Program increased to 10.94 points after $(\mathrm{p}=0.0001)$, which was seen in improvement of gross, medium and fine manual skills, eye-hand coordination and space-time orientation for manual abilities.

Progress in $36.67 \% / 11$ out of 30 reflex patterns showed levels very close to statistical significance and was noted in improvement of sensory-motor integration and motor abilities and skills. 


\section{ATEC test, Sociability, $n=30$}

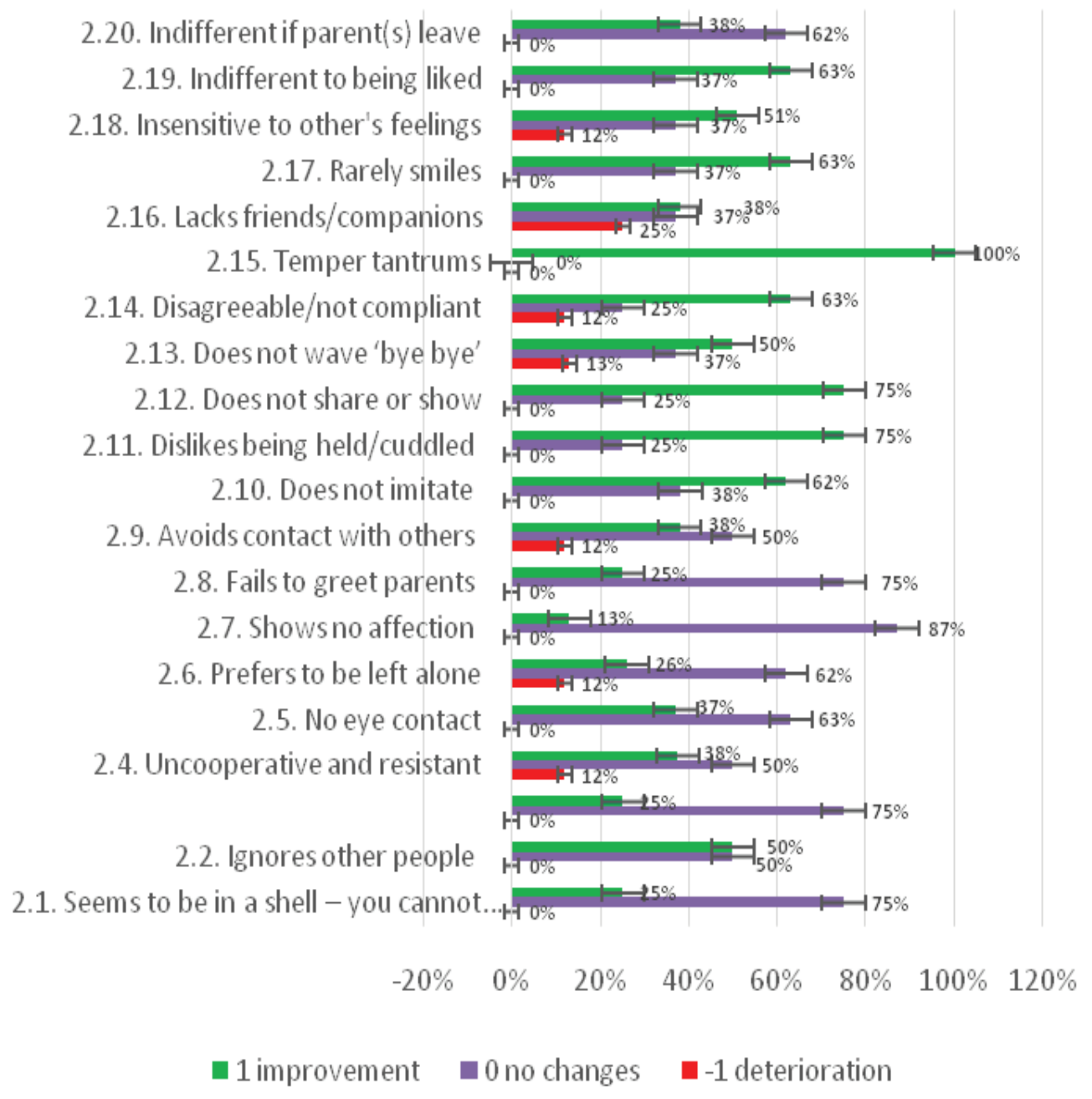

Figure 12: ATEC Questionnaire results: relative distribution of changes in Socializationcategory2 for 30 individuals aged 6-11 years before and after the MNRI intervention.

Qualitative analysis allowed us to group reflex patterns based on their level of influence in different areas of life functioning of children with ASD reflecting progress gained in:

A) Protective responses regulation: Core Tendon Guard and Hands Supporting affected their ability for positive protection and survival, improvement in territorial behavior and ability to meet new people; the Moro and Fear Paralysis reflex patterns allowed for more courage when facing sudden and unexpected stimulus (touch, visual, and auditory) and sudden changes of the body in space.

B) Postural control and cross motor coordination: Automatic Gait, Trunk Extension, Grounding, Symmetrical Tonic Neck (STNR), Spinning, Locomotion, Balancing and Flying and Landing created the basis for positive changes in trunk-head control, eye-head leveling, crawling, matured walking, balance, rotation of body (giving freedom to its horizontal planes); static and dynamic equilibrium.
C) Fine motor coordination-Robinson Hands Grasp, Hands Pulling and Babkin Palmomental created the basis for improvement in manual skills, fine motor, hand-eye coordination, muscle tone regulation in upper limb muscles, communication, speech, visual (convergence-divergence, near-far, 3D vision, peripheral vision), visual-auditory coordination, eating skills, speech and articulation abilities, goal orientation, focusing and stability, head righting, eye tracking, reading, writing, drawing.

D) Motor coordination of the lower limbs-Babinski, Leg Cross Flexion/Extension affected grounding, stability, protection, control of joints, walking balance, cross lateral gross motor programming and control, differentiation of limbs, equilibrium, postural control, and head righting, and focusing.

E) Spinal control-the Spinal Galant and Perez assisted in bettering motor abilities and coordination of the lower limbs. Work with these 


\section{ATEC test, Sociability, $n=30$}

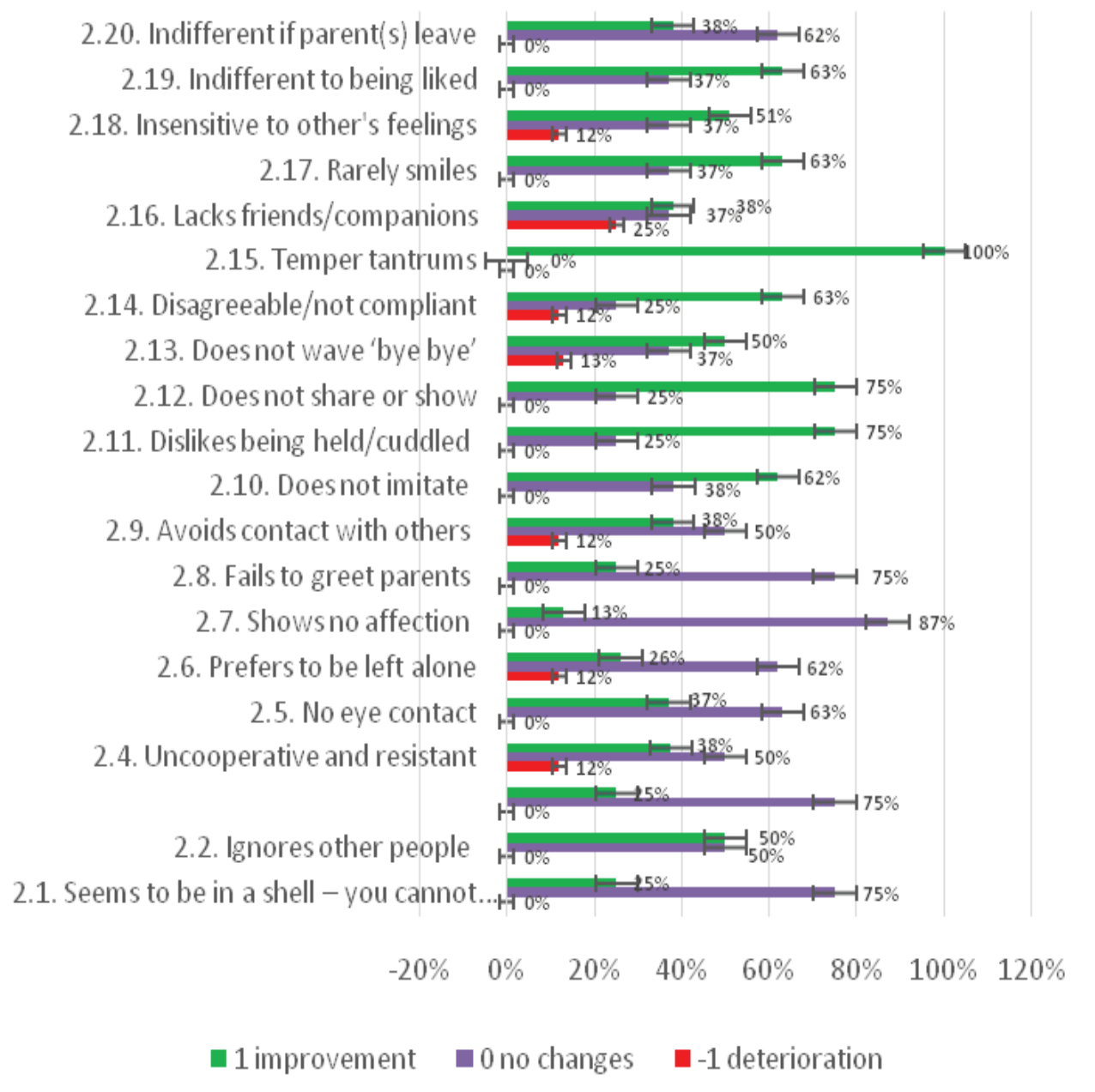

Figure 13: ATEC Questionnaire results: relative distribution of changes in Sensory/Cognitive Awareness category 3 for 30 individuals aged 6-11 years before and after the MNRI intervention.

reflex patterns initiated improvements in tactile sensory sensitivity of the whole body, release of stress hormones and detoxification.

F) Emotional-behavioral regulation-Spinal Perez, Babkin Palmomental, Flying and Landing and Abdominal Sleep Posture regulated the stress hormones release and endorphin increase for improved mood and mental state, ability to calm down-keep inner peace, detoxification, release of anger and frustration, improve sleep pattern.

G) Cognitive abilities-the Pavlov Orientation, ATNR, STNR, Flying and Landing reflex patterns able to lead to positive changes in overall cognition including curiosity, goal orientation, focusing, reading, writing, and drawing.

\section{Progress in results of the brain maps}

Quantitative Brain Mapping-QEEG analysis is based on our knowledge of neurophysiology and the functions of different brain areas. Identifying localized changes using QEEG in the context of behavioral, sensory, or communication symptoms [52] can help to better understand the impact of MNRI neuromodulation therapy on brain activity and its positive changes. Higher brain structures have evolved from the lower, so the action of cortical centers depends on the proper functioning of the subcortical structures $[15,17,61]$.

The use of the Z-score parameter in this study gave the opportunity to compare the QEEG results of the group of children with autism with the normative results [29].

As part of the QEEG quantitative analysis, the change in power spectral density of 10 frequency ranges for 19 leads from the head surface assessing 190 parameters for each patient age group. The obtained QEEG results in the group of children with ASD indicates the normalization of medians of the Z-Score parameter, in particular brain

Citation: Nowak K, Sobaniec P, Sobaniec W, Akhmatova N, Shackleford P (2020) Evaluation of the Therapeutic Effect of MNRI Reflex Neuromodulation on Children Diagnosed with Autism Based on Reflex Assessments, QEEG Analysis and ATEC Questionnaire. J Neurol Neurobiol 


\section{ATEC test \\ Healthy/Physical/Behavior; $n=30$}

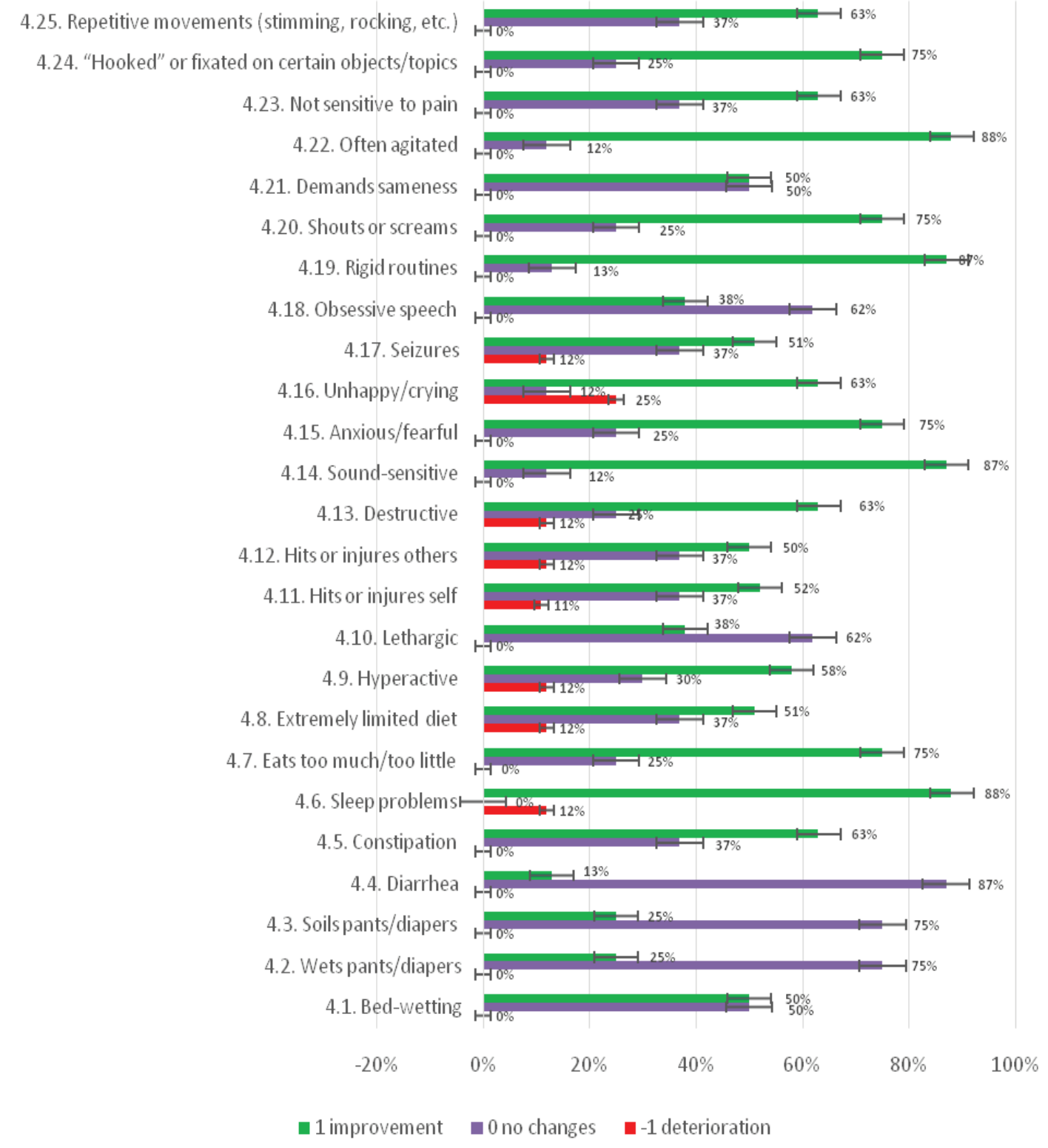

Figure 14: ATEC Questionnaire results: relative distribution of changes in the Physical Health, Behavior category 4 for 30 individuals aged 6-11 years before and after the MNRI therapy.

areas for leads from the frontal region of the brain F4 as well as Fz. The decrease in fast wave values and normalization of EEG recording may correlate with noted changes: more relaxation and greater calmness in these children, also improvement of social interactions, control of emotions and behavior.

The location of $\mathrm{Fz}$ is in the secondary motor cortex, Brodmannn field $6[62]$ and, indirectly, the deep brain structures such as the anterior gyrus. Area 6 is mainly responsible for the activity of proximal muscles and the torso, and damage to this area can cause kinetic apraxia, while the left sensory-motor region participates in the initiation and maintenance of speech. Studies using fMRI have shown that, in individuals with ASD, structural and functional AAC disorders are responsible for rigid and stereotypical behavior and fear of new situations [52].

In our research, normalization of medians of the Z-Score parameter from the central region was also observed. In the age group of younger children, normalization of parameters was visible in the areas $\mathrm{C} 3$, $\mathrm{C} 4$ and $\mathrm{Cz}$. These points are located in the area of the sensory-motor cortex [63]. These areas are also involved in voluntary movements, the organization of movements, and also in the functions of mirror

Citation: Nowak K, Sobaniec P, Sobaniec W, Akhmatova N, Shackleford P (2020) Evaluation of the Therapeutic Effect of MNRI Reflex Neuromodulation on Children Diagnosed with Autism Based on Reflex Assessments, QEEG Analysis and ATEC Questionnaire. J Neurol Neurobiol 6(2): dx.doi.org/10.16966/2379-7150.165 
neurons, which are likely to become active when observing the actions of others. These functions appear to be disturbed in people with ASD. This area connects to the hill and the cerebellum, which is responsible for maintaining balance and correct posture. The cerebellum also participates in the modulation of Reticular Formation (RAS), which stimulates the cerebral cortex to pay attention to new stimulation [26].

Median decrease and normalization of recording were also observed in the temporal region of cortex. The data shows noted improvements in the electrodes registering the waves from the temporal region in areas F8, T3, T4, T6 responsible for reduction of sensory hypersensitivity, especially to sounds and olfactory sensations and explains the reason for improvement in auditory and visual memory and sequencing of events. Record of normalization in these areas of brain maps in these children also resulted in improved abilities for processing complex sounds, generating words and sentences. Also, improvements to this area of the brain known for the executive role of decisions making [21] led to positive changes in their own behavior perception by children in our studies.

Changes in these areas of the brain also were seen in improved functions of such reflexes, as: ATNR, STNR, Hands Pulling, Babkin Palmomental, and also Pavlov Orientation.

Brodmann fields 41 and 42 take part in listening to different frequencies, tones, harmony, intensity of acoustic patterns and volume. Disorders in this area can cause poor or loss of sound awareness.

In summing up the results of studies using QEEG, it is worth emphasizing that Brain Maps of children in this study group, prior and after therapy showed statistical significance in normalization of 41 out of 190 assessed spectral power parameters, 6 parameters showed deterioration, 143 without significant changes. The improvement mainly concerned the frontal, central and temporal leads for various parameters.

Masgutova Neurosensorimotor Reflex Integration is an effective composition of tools for conducting a holistic neurodevelopment therapy for children with autism $[22,33,39,64]$ and other disorders $[19,34,65,66]$. The therapeutic effects of MNRI therapy have been checked using objective independent assessment tools prior and after the 8-day rehabilitation cycle. The MNRI program is scientifically researched and evidence-based, safe and easy in utilization of multiprofiled rehabilitation.

\section{Progress in results of the ATEC test}

In individuals with ASD, a number of common symptoms are seen, including social deficits, communication impairment, and repetitive or stereotyped behavior [67-70]. Some studies of their features found specific correlations: [71]

-Tactile hyperactivity is associated with rigid inflexible behavior

-Repetitive stereotypical images and vocabulary described the importance of hyperactivity of the auditory and visual senses in an increased level of arousal [67] [68]

-Sensory hypersensitivity increased the rate of intrusive behavior

-Hyperactivity and sensory hypersensitivity affect an increased level of anxiety in both children with autism as well as other neurological and psychiatric disorders [69]

-The exorbitant sympathetic arousal in response to normal levels of sensorimotor stimulation predicts sleep disorders in children with autism. Similarly, the excessive response in behavioral patterns may correlate with sleep problems [70].

The ATEC questionnaire, that contains symptom and features of ASD, was completed by the parents/guardians of their children prior to and immediately after the MNRI rehabilitation.

The results of this study indicate that changes in children with ASD aged 6-11 years were reported concerning:

Category 1: Speech, language and communication [Figure 11]. Parents of $31 \%$ of participants noted changes (statistically significant level), of $31 \%$-close to statistical significance, and parents of $33 \%$ of them did not note changes in this category; unclear evaluations were given for $5 \%$ of children. However, the main achievements in this category are increases in non-verbal and verbal communication, better focusing, increased cognition (responding properly to their own names-38\%) and to the word "No" (38\%).

Category 2: Socialization [Figure 12]. Parents of $48 \%$ of participants noted changes (statistically significant) of $22 \%$-close to statistical significance, and parents of $25 \%$ of them did not note changes in this category; unclear evaluations were given for $5 \%$ of children. The main achievement in this category shows greater changes in reduction of tantrums in all participants, increased imitation in $61 \%$ of respondents, increased the desire to hug in $68 \%$ of children, as well as more sharing property in $68 \%$ of children.

Category 3: Sensory Sensation and Cognitive Awareness [Figure 13]. Parents of $38 \%$ of participants noted changes (statistically significant) of $16 \%$-close to statistical significance, and parents of $36 \%$ of them did not note changes in this category; unclear evaluations were given for $10 \%$ of children. The main achievement in this category is greater changes in responses/attention to praising (62\% children), the appropriate use of toys $(62 \%)$, and proper emotional and facial expressions in $62 \%$ of subjects.

Category 4: Sensory/Cognitive Awareness [Figure 14]. Parents of $59 \%$ of participants noted changes (statistically significant) of $10 \%$-close to statistical significance, and parents of $27 \%$ of them did not note changes in this category; unclear evaluations were given for $4 \%$ of children. The main achievements in this category are the greater changes in responses/attention to praising (62\% children), the appropriate use of toys (62\%), and proper emotional and facial expressions in $62 \%$ of subjects. The detailed analysis shows that parents noted improvement in $88 \%$ of children with ASD (after the MNRI program) in sleeping patterns-the quality and length of sleep, in $87 \%$-reduction of auditory sensitivity, and in 85\%-improvement in psychomotor silence/relaxation, reduction of frustration and agitation as well as of rigid patterns and behaviors, in $62 \%$ of children-the decrease of tactile and sonic sensitivity, in 63\%-definite improvement in the work of the gastrointestinal tract, and in $72 \%$-decrease of constipation and normalization in eating (regularity, quantity and choice of food).

\section{Summary}

The MNRI Program demonstrates the neuromodulative and normalization effects on brain wave spectrum (QEEG), supports the development of reflex patterns that result in the development of some very challenging areas of life and learning of children with ASD-speech and communication, socialization, sensory and cognitive awareness, physical health and behavioral-emotional regulation. In MNRI the concept of restorative techniques for dysfunctional reflex circuits is utilized. MNRI corrective work allows paving and rerouting of nerve pathways to bring them into a state of proper 
neurophysiological functioning in order to improve the balance of the excitation-inhibition processes of the nervous system, increase stress resilience and neuroplasticity.

The results of the presented study indicate that the neurodevelopment of individuals with ASD-corrective and preventive-can be enhanced with this unique novel form of MNRI neuromodulation treatment.

The goal of this study-the evaluation of effects of an intensive MNRI rehabilitation treatment included:

- MNRI Reflex Assessment (Reflex Maturity Scaling)

- Brain mapping-a qualitative analysis of EEG

- The social-behavioral-cognitive traits ATEC test by parents or caregivers for evaluation of the effect of interventions on their children with the ASD

- The MNRI Reflex sensory-motor neuromodulation rehabilitation program by Dr. Sechenov IM. [17].

Thirty children with ASD aged 6-11 years were participants of the 8-day MNRI Reflex Integration intervention.

All three evaluation methods showed substantial positive changes in children with ASD in several important developmental areas after the MNRI treatment, particularly:

\section{Reflex Pre-and Post-Assessment}

Significant progress in $33.33 \% / 10$ out of 30 patterns took place on a statistical level ( $p>0.01$ ), and in $36.67 \% / 11$ patterns on close to statistical significance, which is supported by qualitative analyses.

This study shows that the neurodevelopment and overall functioning of individuals with neurodeficits such as ASD is not static in pathologies and could be successfully improved with the MNRI novel form of neuromodulation treatment.

\section{QEEG}

$76.67 \%$ (23 out of 30 ) children displayed a reorganization of spontaneous brain electrical activity observed in the increase in alpha frequency range and decrease in fast beta activity in parietal and temporal locations. This may be connected with the positive and stable therapeutic effect of MNRI on motor disorders originating in the central nervous system.

\section{The ATEC test demonstrated that parents noticed changes in:}

Category 1: Speech, language and communication-changes were noted in $31 \%$ of children (statistically significant level) and in $31 \%$ by a close to statistical significance. The post-test results show that parents noted increases in abilities of non-verbal and verbal communication, better focusing, increased proper responses to their own name, and to understanding the word "No"

Category 2: Socialization-changes were noted in $48 \%$ of children (statistically significant level), in $22 \%$ by a close to statistical significance. The main achievement in this category were a reduction of tantrums in all autistic participants and increased imitation abilities, to desire hugs, and share property.

Category 3: Sensory Sensation and Cognitive Awareness-changes were noted in $38 \%$ of participants (statistically significant level), of $16 \%$ by a close to statistical significance. The main achievements in this category are increased abilities to be responsive to praising, use toys appropriately, and use appropriate emotional and facial expressions.

Category 4: Sensory Sensation and Cognitive Awarenesschanges were noted in 59\% of participants (statistically significant level), of $10 \%$ by a close to statistical significance. Improvements in sleeping pattern, reduction of auditory sensitivity, improvement in psychomotor silence/relaxation, reduction of frustration and agitation, as well as of rigid patterns and behaviors, tactile and sonic sensitivity, gastrointestinal tract difficulties, and constipation and normalization in eating were observed. MNRI facilitates neurodevelopment, stress resiliency, neuroplasticity, and optimal learning opportunity.

\section{Funding Source}

This work was supported by the Bridge to Healing, a 401(c) 3 organization.

\section{Conflict of Interest}

The authors have no conflict of interest.

\section{Acknowledgments}

Dr. Svetlana Masgutova for sharing her expertise on Reflex Assessment and the sMNRI Therapy Programs used in this study.

Parents for participation in the study with their children with ASD for giving us the unique possibility to evaluate the neuromodulative effect of MNRI and help many thousands of children with ASD and other neurodeficits.

Tatiana Tatrinova for her long-time collaboration and support with editing this MNRI publication.

Sally Averkamp for her long-time collaboration and technical editing of this paper.

Tricia Borsh, the Founder of the Bridge-to-Healing Foundation for subsidizing MNRI costs.

Professor J. Lukas Koberda, Tallahassee Neuro-Balance Center, for the QEEG part of the research and results interpretation.

Adrian E. Quevedo, MS, for recording the QEEGs and creating reports. All those who were helping with this research.

\section{References}

1. Green SA, Rudie JD, Colich NL, Wood JJ, Shirinyan D, et al. (2013) Overreactive brain responses to sensory stimuli in youth with autism spectrum disorders. J Am Acad Child Adolesc Psychiatry 52: 1158-1172.

2. Zwaigenbaum L (2010) Unique developmental differences associated with ASD. Autism 14: 5-7.

3. Kanner L (1973) The birth of early infantile autism. J Autism Child Schizophr 3: 93-95.

4. Rybakowski F, Bialek A, Chojnicka I, Dziechciarz P, Horvath A, et al. (2014) Autism spectrum disorders-epidemiology, symptoms, comorbidity and diagnosis. Psychiatr Pol 48: 653-665.

5. Pisula E (2010) Autyzm: Przyczyny, symptomy, terapia. Gdańsk: Wydawnictwo Harmonia, Poland.

6. Cox A, Klein K, Charman T, Baird G, Baron-Cohen S, et al. (1999) Autism spectrum disorders at 20 and 42 months of age: stability of clinical and ADI-R diagnosis. J Child Psychol Psychiatry 40: 719-732.

7. Allen MC, Capute AJ (1986) The evolution of primitive reflexes in extremely premature infants. Pediatr Res 20: 1284-1289.

8. Silva LM, Schalock M, Ayres R, Bunse C, Budden S (2009) Qigong massage treatment for sensory and self-regulation problems in young children with autism: a randomized controlled trial. Am J Occup Ther 63: 423-432. 
9. French LR, Bertone A, Hyde KL, Fombonne E (2013) Epidemiology of Autism Spectrum Disorders. Neurosci Autism Spectr Disord 3-24.

10. Tordjman S, Najjar I, Bellissant E, Anderson GM, Barburoth M, et al. (2013) Advances in the research of melatonin in autism spectrum disorders: literature review and new perspectives. Int J Mol Sci 14: 20508-20542.

11. Banasiak M, Witusik A, Pietras T, Górski P (2010) Epidemiologia autyzmu. In: Pietras T, Witusik A, Gałecki $P$ (eds) Autyzmepidemiologia, diagnoza i terapia. Wrocław, Poland.

12. Geier DA, Kern JK, Geier MR (2013) A Comparison of the Autism Treatment Evaluation Checklist (ATEC) and the Childhood Autism Rating Scale (CARS) for the Quantitative Evaluation of Autism. J Ment Health Res Intellect Disabil 6: 255-267.

13. Shackleford P (2015) Svetlana Masgutova Educational Institute ${ }^{\circ}$ for Neuro-Sensory-Motor and Reflex Integration, SMEI (USA).

14. Akhmatova NK, Masgutova SK, Shubina IZ, Akhmatov EA, Khomenkov VV, et al. (2015) Immunological Effects of Masgutova Neurosensorimotor Reflex Integration in Children with Recurrent Obstructive Bronchitis. Int J Neurorehabilitation Eng 2: 2-9.

15. Pavlov IP (1927) Conditioned Reflexes: An investigation of the physiological activity of the cerebral cortex. In: Anrep GV (eds) Oxford University press, United Kingdom.

16. Sechenov I (1965) Reflexes of the brain. In: Gibbons G (eds) MIT Press, United States.

17. Sechenov IM (1995) Physiology of behavior. Scientific Works. In: Yaroshevsky MG (eds) Moscow, Russia.

18. Sherrington SCS (1947) The Integrative Action of the Nervous System. Cambridge University Press, United Kingdom.

19. Nowak K, Sendrowski K (2016) Neurophysiological Aspects of NeuroTactile Therapy of Masgutova Neurosensory Motor Reflex Integration MNRI Method. Med Rehabil 20: 1-10.

20. Marieb EN, Hoehn K (2012) Human Anatomy \& Physiology. $9^{\text {th }}$ Edition, San Francisco, CA: Pearson Education.

21. Nuwer MR (2003) Clinical use of QEEG. Clin Neurophysiol 114: 2225.

22. Deiss T, Meyers R, Whitney J, Bell C, Tatarinova T, et al. (2019) Physiological Markers and Reflex Pattern Progression in Individuals with Neurodevelopmental Deficits Utilizing the MNRI Method. Neurosci Med 10: 30-54.

23. Diagnosis. J Child Adolesc Psychiatr Psychol 40: 719-732.

24. Prichep LS, John ER (1992) QEEG profiles of psychiatric disorders. Brain Topogr 4: 249-257.

25. Artemowicz B, Sobaniec P (2011) EEG investigation in neuropediatrics. Neurol Dziec 41: 89-95.

26. Matusevich D, Ruiz M, Vairo MC (2002) QEEG and brain mapping. Historial develoment, clinical practices and epistemological issues. Vertex 13: 198-204.

27. Tatum WO, Olga S, Ochoa JG, Munger Clary H, Cheek J, et al. (2016) American Clinical Neurophysiology Society Guideline 7: Guidelines for EEG Reporting. J Clin Neurophysiol 33: 328-332.

28. Tsuchida TN, Acharya JN, Halford JJ, Kuratani JD, Sinha SR, et al. (2016) American Clinical Neurophysiology Society: EEG Guidelines Introduction. Neurodiagn J 56: 231-234.

29. NeuroGuide ${ }^{\mathrm{TM}}$ by Applied Neuroscience, Inc.
30. Rimland B, Edelson SM (1999) Autism Treatment Evaluation Checklist (ATEC). Autism Research Institute, San Diego, CA.

31. Al Backer NB (2016) Correlation between Autism Treatment Evaluation Checklist (ATEC) and Childhood Autism Rating Scale (CARS) in the evaluation of autism spectrum disorder. Sudan J Paediatr 16: 17-22.

32. Nowak K, Muzaj H, Nawrocka H, Sobaniec P (2018) Evaluation of the effectiveness of MNRI therapy (Masgutova Neurosensorimotor Reflex Integration) in children with autism based on the ATEC (Autism Treatment Evaluation Checklist) test-a preliminary report. Med Rehabil 22: 20-34.

33. Koberda JL, Akhmatova N, Akhmatova E, Bienkiewicz A, Nowak K, et al. (2016) Masgutova Neurosensorimotor Reflex Integration (MNRI) Neuromodulation Technique induces Positive Brain Maps (QEEG) Changes. J Neurol Neurobiol 2.

34. Pilecki W, Masgutova S, Kowalewska J, Masgutov D, Akhmatova $\mathrm{N}$, et al. (2012) The impact of rehabilitation carried out using the Masgutova Neurosensorimotor Reflex Integration method in children with cerebral palsy on the results of brain stem auditory potential examinations. Adv Clin Exp Med 21: 363-371.

35. Renard-Fontaine I (2017) Effect of Reflex Neuromodulation on an Infant with Severe Amniotic Band Syndrome: A Case Report on the use of MNRI Techniques for Physical Therapy. Int J Neurorehabilitation 4: 248.

36. Nowak K, Sobaniec P, Muzaj H, Łotowska JM (2018) Znaczenie neurosensomotorycznej terapii MNRI wg dr S Masgutovej $\mathrm{u}$ pacjentów z zaburzeniami neurologicznymi. Neurol Dziec 54: 37-52.

37. Masgutova SK, Akhmatova NK, Sadowska L, Shackleford P, Akhmatov EA (2016) Neurosensorimotor Reflex Integration for Autism: a New Therapy Modality Paradigm. J Pediatr Neurol Disord 2: 1000107.

38. Akhmatova N, Akhmatova E (2016) Influence of MNRI on the Immune Status of Children with Down Syndrome. J Clin Cell Immunol 8: 1-8.

39. Masgutova S, Masgutov D, Lieske T (2018) Effects of MNRI Visual Reflex Neuro-Training on Visual and Academic Skills of Children with Autism. Int J Sch Cogn Psychol 5: 1-15.

40. Masgutova S, Masgutov D (2015) Parents' guide to MNRI: The Masgutova Neurosensorimotor Reflex Integration-MNRI ${ }^{\circ}$ Method. Svetlana Masgutova Educational Institute.

41. Ortego L, Pelican E, Callaba L, Marks T (2015) An Investigation of the Effects of $\mathrm{MNRl}^{\circ}$ Techniques on the Educational Performance of Kindergarten Students. SMEI 142-150.

42. Masgutova S, Akhmatova N, Ludwika S (2016) Reflex Profile of Children with Down Syndrome Improvement of Neurosensorimotor Development Using the $\mathrm{MNRI}^{\circ}$ Reflex Integration Program. Int J Neurorehabilitation 3: 197.

43. Masgutova S, Regner A $(2008,2011)$ Language Development Using Sensory-Motor Integration Approach. MISM, Wroclaw: 167.

44. Sadowska L (2008) Wroclaw Rehabilitation Model (WRM) in early diagnosis and therapy in children with Down syndrome. In: Kaczmarek B (eds) Facilitation of Development of Children with Down Syndrome-Theory and Practice. Krakow, Impuls Kraków, Poland 195-224.

45. Miller BL, Cummings JL (2017) The Human Frontal Lobes: Functions and Disorders. $2^{\text {nd }}$ Edition, The Guilford Press, USA.

46. Barbey AK, Koenigs M, Grafman J (2013) Dorsolateral prefrontal contributions to human working memory. Cortex 49: 1195-1205.

Citation: Nowak K, Sobaniec P, Sobaniec W, Akhmatova N, Shackleford P (2020) Evaluation of the Therapeutic Effect of MNRI Reflex Neuromodulation on Children Diagnosed with Autism Based on Reflex Assessments, QEEG Analysis and ATEC Questionnaire. J Neurol Neurobiol 6(2): dx.doi.org/10.16966/2379-7150.165 
47. Head H, Holmes G (1911) Sensory Disturbances from Cerebral Lesions. Brain 34: 102-254.

48. Graziano M (2008) The Intelligent Movement Machine. Oxford, UK: Oxford University Press.

49. Elliott R (2003) Executive functions and their disorders. Br Med Bul 65: 49-59.

50. Monsell S (2003) Task switching. Trends in Cognitive Sciences 7: 134140

51. Chan RC, Shum D, Toulopoulou T, Chen EY (2008) Assessment of executive functions: review of instruments and identification of critical issues. Arch Clin Neuropsychol 23: 201-216.

52. Conson M, Errico D, Mazzarella E, Giordano M, Grossi D, et al. (2015) Transcranial Electrical Stimulation over Dorsolateral Prefrontal Cortex Modulates Processing of Social Cognitive and Affective Information. PLoS One 10: e0126448.

53. Thatcher RW (2012) Coherence, phase differences, phase shift, and phase lock in EEG/ERP analyses. Dev Neuropsychol 37: 476-496.

54. Brodmannn K (1909) Kapitel in Vergleichende Lokalisationslehre der Grosshirnrinde (Leipzig: Verlag von Johann Ambrosias Barth) Beschreibung der einzelnen Hirnkarten IV.

55. Krizman J, Skoe E, Krausa N (2010) Stimulus Rate and Subcortical Auditory Processing of Speech. Audiol Neurootol 15: 332-342.

56. Lu MT, Preston JB, Strick PL (1994) Interconnections between the prefrontal cortex and the premotor areas in the frontal lobe. J Comp Neurol 341: 375-392.

57. Gerloff C, Corwell B, Chen R, Hallett M, Cohen LG (1997) Stimulation over the human supplementary motor area interferes with the organization of future elements in complex motor sequences. Brain 120: $1587-1602$

58. Harmony T (2013) The functional significance of delta oscillations in cognitive processing. Front Integr Neurosci 7: 83.

59. Haag G, Botbol M, Graignic R, Perez-Diaz F, Bronsard G, et al. (2010) The Autism Psychodynamic Evaluation of Changes (APEC) scale: a reliability and validity study on a newly developed standardized psychodynamic assessment for youth with Pervasive Developmental Disorders. J Physiol Paris 104: 323-336.
60. Piaget J (1974) The future of developmental child psychology. J Youth Adolesc 3: 87-93.

61. Vygotsky L (1986) Child psychology. The problems of child development. Pedagogika 64: 109-119.

62. Crinion J, Turner R, Grogan A, Hanakawa T, Noppeney U, et al. (2006) Language Control in the Bilingual Brain. Science 312: 1537-1540.

63. Hart HC, Palmer AR, Hall DA (2004) Different areas of human nonprimary auditory cortex are activated by sounds with spatial and nonspatial properties. Hum Brain Mapp 21: 178-190.

64. Masgutova S, Sadowska L, Kowalewska J, Masgutov D, Akhmatova N, et al. (2016) Use of a Neurosensorimotor Reflex Integration Program to Improve Reflex Patterns of Children with Down Syndrome. J Neurol Neurosci 6: 4

65. Masgutova S (2016) Post-Trauma Recovery in Children of Newtown, CT using MNRI Reflex Integration. J Trauma Stress Disor Treat 6: 5.

66. Masgutova S, Akhmatova N, Sadowska L, Shackleford P, Akhmatov E (2016) Progress with Neurosensorimotor Reflex Integration for Children with Autism Spectrum Disorder. J Neurol Psychol 4: 14.

67. Boyd BA, McBee M, Holtzclaw T, Baranek GT, Bodfish JW (2009) Relationships among Repetitive Behaviors, Sensory Features, and Executive Functions in High Functioning Autism. Res Autism Spectr Disord 3: 959-966.

68. Liss M, Saulnier C, Fein D, Kinsbourne M (2006) Sensory and attention abnormalities in autistic spectrum disorders. Autism 10: 155-172.

69. Pfeiffer B, Clark GF, Arbesman M (2018) Effectiveness of Cognitive and Occupation-Based Interventions for Children with Challenges in Sensory Processing and Integration: A Systematic Review. Am J Occup Ther 72: 7201190020p1-7201190020p9.

70. Reynolds S, Glennon TJ, Ausderau K, Bendixen RM, Kuhaneck HM, et al. (2017) Using a Multifaceted Approach to Working With Children Who Have Differences in Sensory Processing and Integration. Am J Occup Ther 71: 7102360010p1-7102360010p10.

71. Baranek GT, Foster LG, Berkson G (1997) Tactile defensiveness and stereotyped behaviors. Am J Occup Ther 51: 91-95.

Citation: Nowak K, Sobaniec P, Sobaniec W, Akhmatova N, Shackleford P (2020) Evaluation of the Therapeutic Effect of MNRI Reflex 